\title{
OFFICIAL USE
}

\section{KNOLLS ATOMIC POWER LABORATORY}

\author{
Operated for the \\ U.S. ATOMIC ENERGY COMMISSION
}

Semiahnual Progress Report

of

DEVELOPMENT ACTIVITIES

IN THE

HEALTH PHYSICS DIVISION

Submitted by

R. Z. Bouton and R. W. Houston

Knolls Atomic Power Laboratory and

W. Van der Grinten

Research Laboratory, General Electric Co.

\section{January 1951}

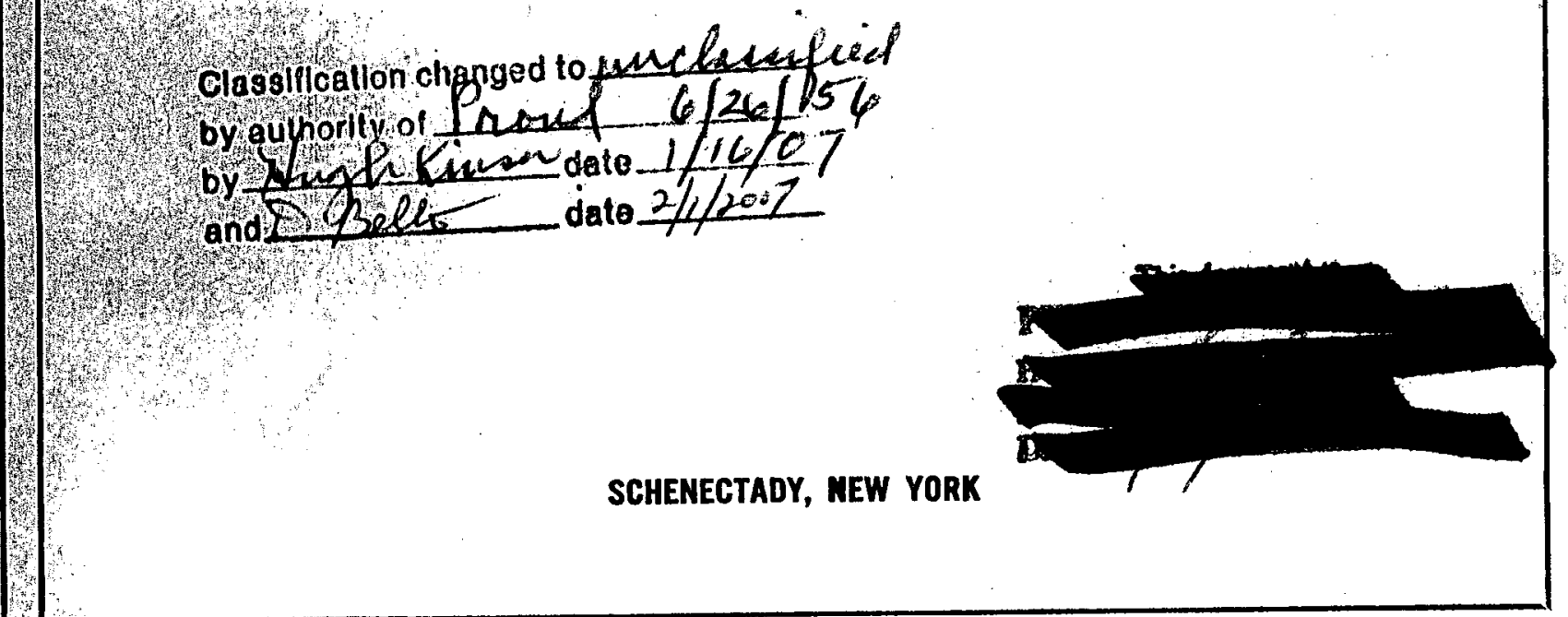




\author{
REPORT NO. KAPL-492 \\ 1 \\ Health and Biology \\ General Electric Company \\ KNOLIS ATOMIC POWER LABORATORY \\ Schenectady, New York \\ Semiannual Progress Report \\ of \\ DEVELOPMENT ACTIVITIES \\ IN THE \\ HEALTH PHYSICS DIVISION \\ January 1951 \\ Submitted by \\ Photostat Price $\$ 1.80$
Microfilm Price $\$ 30$
Available from the
Office of Technical Services
Department of Commerce
Washington 25, D. C. \\ R. Z. Bouton and R. W. Houston \\ Knolis Atomic Power Laboratory \\ and \\ W. Van der Grinten \\ Research Laboratory, General Electric Co.
}

\author{
Operated for the \\ United States Atomic Energy Commission \\ by the \\ General Electric Company \\ Contract No. W-31-109 Eng-52
}

\title{
LEGAL NOTICE
}

This report was prepared as an account of Government sponsored work. Neither the United States, nor the Commission, nor any person acting on behalf of the Commission:

A. Makes any warranty or representation, express or implied, with respect to the accuracy, completeness, or usefulness of the information contained in this report, or that the use of any information, apparatus, method, or process disclosed in this report may not infringe privately owned rights; or

B. Assumes any liabilities with respect to the use of, or for damages resulting from the use of any information, apparatus, method, or process disclosed in this report.

As used in the above, "person acting on behalf of the Commission" includes any employee or contractor of the Commission to the extent that such employee or contractor prepares, handles or distributes, or provides access to, any information pursuant to his employment or contract with the Commission. 


\section{DISCLAIMER}

This report was prepared as an account of work sponsored by an agency of the United States Government. Neither the United States Government nor any agency Thereof, nor any of their employees, makes any warranty, express or implied, or assumes any legal liability or responsibility for the accuracy, completeness, or usefulness of any information, apparatus, product, or process disclosed, or represents that its use would not infringe privately owned rights. Reference herein to any specific commercial product, process, or service by trade name, trademark, manufacturer, or otherwise does not necessarily constitute or imply its endorsement, recommendation, or favoring by the United States Government or any agency thereof. The views and opinions of authors expressed herein do not necessarily state or reflect those of the United States Government or any agency thereof. 


\section{DISCLAIMER}

Portions of this document may be illegible in electronic image products. Images are produced from the best available original document. 


\section{UNCLASSIFIED}

KAPL-492

CONTENTS

Page

Preface ........................ 6

Introduction ...................... 7

Summary of Work Accomplished ............... 7

Air Sampling Studies ................... 9

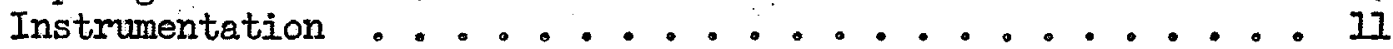

Size Distribution of KAPL Shop Dusts . . . . . . . . 27

Aerosol Filter Tests ............... . . 34

Tritium Determination in Aqueous Samples ........... 43

Fluorimetric Analysis for Beryllium . . . . . . . . . . 54

Low-Level Alpha Measurements . . . . . . . . . . 58

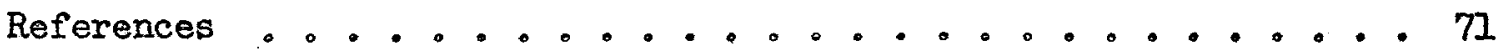




\title{
UNCLASSIFIED
}

\author{
PREFACE
}

This is the first complete progress report of development activities of this division. It covers, therefore, a period somewhat longer than the previous six months and in some parts includes background material which does not necessarily represent experimental progress of this group. The inclusion of such material has been deemed necessary for proper orientation of the work described.

Work on the air sampling studies and the tritium analysis have been the two principal components of the program of the Development Section of this division. The work reported represents approximately $80 \%$ of the time of one laboratory technical staff member and two laboratory assistants. Work on the beryllium analysis is under the cognizance of the Laboratories Section of the division and represents approximately 5\% of the time of one technical staff member and one laboratory assistant. The low-level alpha measurements program has been a cooperative effort with contributions from both of the aforementioned sections and largely under the direction of Dr. W. Van der Grinten of the General Electric Research Laboratory. 


\author{
SUMARY
}

\title{
Introductigs
}

This reprt sumarizes the progress in developmental activities in the Jealth Figsios Dirision of the Knolls Atomic Power Laboratory for the period ending Deenter 31, 2950. Development work on a new method of sampling aerosols for the deternination of conentration and particle size distribution I: included wder the general heading of "Air Samping Studies." The method is being apolisd in a study of the size distribution of KAPL shop dusts and in $a$ proprifiatic research proben on the efficiency of various filter media for asrosel sampling.

Progress is also reported on a nuclear counter technique for the determination of tritium as Ho in urine or other aqueous samples, investigation. of the morin flumime ric analysis for beryllium, and a ruclear filim procedure fort the determination of very low levels of alpha activity.

\section{Summary of york Acomplished}

A majer portion of the effort ba air sapling studies has been devoted to the development of a modified electrostatic precipitator. Utilizing a point-to-plane electrode configuration, the entire aerosol deposit is obtained on a halfwinch diameter surface. This is removed quantitatively in a transparent plastio film and may undergo chemical analysis or microscopic size count. Additional data are necessary to validate this method of campline and the subsequent size-counting technique.

Preliminary results on size distribution of KAPL shop dusts have been obtained utilizing the modified electrostatic preeipitator. Without differentiating between uranjum, beryllium, or other nuisance dusts which appear opaque in the light microscope, the indicated mass median diameters are on the order of $1.5 \mu$ with no material larger than about $4.0 \mu$ being observed.

The design and construction of the aerosol filter test facilities have been completed. These provide for the generation of a metalic dust similar in nature to that found in the "potentially hazardous" areas of this laboratory. Frovisions for the regulation of the upper size and concentration limits have been incorporated. Whe unit also provides for uniform dispersion of the aerosol into a lerge diameter chamber and for jokinetic sampling through any of four ports.

Pesta to determine the aeroscl filtration efficiencies of two types of filter papers as functions of particle size and fow rate are about to begin.

As a dixect result of the interest of this division in aerosol problems, it has been possible to give assistanee to the Chemical. Engineering Division. of this laboratory in sampling problems connected with the evaluation of the

\section{UNCLASSIFIED}


high-level radioactive solid waste incinerator. The results of these studies will be reported elsewhere.

A method has been developed for low-level determinations of tritium as HTO in urine or other water samples. Tritiated methane is generated by the reaction of the sample on aluminum carbide. The partially purified gas is pumped into a counter tube to a pressure of 0.5 at and counted with a proportional alpha counter. The sensitivity of the procedure is better than $1 \mu c$ per liter of aqueous sample. Although the method has been adapted to routine urinalysis, it is undergoing continued investigation.

The interference of certain cations on the results of fluorimetric analysis for beryllium has been investigated. To take advantage of the sensitive range, $0.001-0.050 \mu \mathrm{g} \mathrm{Be} / \mathrm{ml}$, these cations or their effects must be eliminated. Work is progressing in this direction. Some assistance has been given groups at Los Alamos and at $M_{0} I_{0} T_{0}$ in the application of the method.

Work has progressed on the adaptation of a nuclear film technique, sensitive to 0.01 alphas per $\mathrm{cm}^{2}$ per hour, to health-physics problems; plutonium analysis in urine is of particular interest. Causes of a variable background due to processing of urine samples have been investigated, and it has been shown that plutonium may be detected with the above sensitivity by means of range measurements even with a variable background. 
DEVELOPMENT ACTIVITIES

IN THE

HEALTH PHYSICS DIVISION

Semiannual Progress Report

January 1951

\section{AIR SANPLING STUDIES}

The field of health physics includes problems arising from the escape of radioactive and toxic materials into the atmosphere which subsequently may become health hazards to personnel within and outside of the working areas. In many cases these materials are in the form of particulate matter. The proper evaluation of such hazards covers a wide range of important factors, including the characteristics of the dispersed material, the concentration in air, and the related physiological significance. Adequate determination of these factors depends to a large extent on the sampling and analytical methods employed.

The importance attached to sampling methods is perhaps greater here than in many other problems in chemistry and engineering. Scientific knowledge of inhomogeneous systems has advanced much more rapidly where the inhomogeneity is on a molecular or smaller scale, or on a macro scale. The dispersion of solid or liquid materials in gases falls into the spectrum between these extremes, and one may not always be certain what is meant by homogeneity or inhomogeneity。

Such systems have become known by many different names which usually indicate differences in the characteristics of the disperse phase. Two of the important characteristics in this respect are state of aggregation, i.e.s Iiquid or solid, and "size." Other properties of the dispersoid being constant, the "size" determines the stability of the dispersion in a gravitational field. The term "aerosol" is commonly applied to disperse systems in air in which the earth's gravitational field has little or no effect. The terms dust, fog, spray, and mist are usually used where the particle size extends into the region where gravitational effects are significant.

Toxic or radioactive dispersoids in air cannot be evaluated completely without a knowledge of the size spectrum involved. Any means of sampling atmospheres for such an evaluation should therefore not discriminate against any size range or any other property of the material.

This general subject has received considerable attention in recent years and has resulted in a wide variety of sampling methods. $1-7$ Frequently the application is highly specific, 7 and most of these methods do not yield all of the information that might normally be desired. Some yield results only in terms of mass, number, or activity concentration without reference to size, 
while others may yield only size information and that may be limited. Hatch ${ }^{3}$ has outlined many of the qualifications of a good sampling device for the "health hazard" problem and discusses the relative merits of the best devices now in use, including (1) the cascade impactor, (2) the midget impinger, (3) the glass spiral, and (4) the thermal precipitator. It is significant to note that these devices are in the class of portable instruments for field use as well as for use in the laboratory. Much excellent work was carried out during World War II on precision instruments for analyzing aerosols, e.g., by LaMer, 4 Sinclair, and others. 2 In general, these are not portable instruments, they require extensive calibration procedures for nonuniformly-sized aerosols, and they may have limitations with respect to the lower limit of detectable particle size.

A review of the aforementioned and other literature in this field thus revealed the following information:

I. There does not appear to be any one device or method which is entirely satisfactory for the sampling and characterization of toxic or radioactive air-borne dispersoids.

2. There is insufficient knowledge of the limitations of most sampling methods.

3. Precision laboratory instruments for the complete evaluation of sampling methods are not generally available.

It should be added that these remarks are particularly applicable when the dispersion under study is very dilute and when the characterization should include all particle sizes that may be significant to the basic problem of interest.

Hatch (loc. cit.) has stressed the low concentration characteristic, which is particularly important for radioactive materials. Although inhalation is not the only means by which dusts and aerosols may become health hazards, it is probably the most important. Industrial hygienists have made significant studies of the relationship between particle size and retention in the respiratory system. Brown et $a^{8}$ present some new and interesting data on this subject which should be of considerable significance in the evaluation of toxicological data. However, it does not serve to eliminate the necessity for high efficiency sampling over the entire size spectrum existing. On the contrary, these studies and biological studies on the relationship between toxicity and particle size point up the desirability of more detailed knowledge of size spectra, extending down to the . limits of resolution of the electron microscope.

With these facts in mind, it appeared desirable to undertake a developmental program aimed at a better method of sampling for obtaining all desirable characteristics of airoborne particulate materials. These include the following: (1) mass or activity concentration in air, (2) particle size spectrum, (3) particle shape, (4) nature and extent of aggregation, (5) interrelationship of above characteristics. 
Instrumentation

In general, two different procedures could be followed to derive the information desired: (1) quantitative removal of the disperse phase from air followed by observation and analysis and (2) direct observation and analysis in the air-borne state; i.e., without deposition. In some cases a combination of the two procedures may be desirable.

The particle size spectrum of a given dust or aerosol might be determined (I) by direct measurement at high magnification, or (2) by measurement of some property of the dispersoid which is a known function of particle size. Properties which might thus be measured include rate of motion in (a) a gravitational

field, (b) a centrifugal field, (c) a thermal gradient, (d) an electrostatic field, as well as (e) rate of diffusion due to Brownian motion, (f) optical properties, $(\mathrm{g})$, surface properties, and $(\mathrm{h})$ chemical properties. Although relatively few are sufficiently well understood to serve as the basis for the determination of the particle size spectrum of any aerosol, particularly when the aerosol is of very low concentration and may contain large quantities of "foreign" material.

A large majority of sampling devices make use of a centrifugal force field, e.g.g the cascade impactor, impingers, glass: spiral, etc. These suffer one or more of the following disadvantages:

1. Collection efficiency is low for submicron sizes, making it necessary to add other devices in series.

2. High impact velocities may shatter friable materials and tear apart aggregates.

3. Precise calibration is difficult.

4. Total deposit is divided into several fractions according to size.

It is readily apparent that these four items are closely interrelated. Separation into various size fractions would normally be considered advantageous if precise calibration of the scheme were practicable. It might not always be clear, however, to what extent overlapping would occur without some previous knowledge of the over-all size spectrum of a given sample, Furthermore, the performance of the first stage could certainly never be "calibrated" for a completely unknown dispersoid. Inasmuch as size-counting techniques are normally rather tedious, it would seem desirable not to separate a given sample into several fractions but to incorporate the entire sample in one deposit. These remarks are not applicable, however, in biological and engineering studies where some degree of control exists over the generation and dispersion of a test material. 
Thermal forces have been rather widely used as a means of efficient separation for sampling. 3 With one exception 14 instruments thus far devised use an extremely low flow rate. It is questionable whether or not a representative sample can be obtained drawing only a few $c c$ per minute. There also appears to. be some question about the upper size limit for quantitative collection.

Electrostatic forces have been utilized for both highly efficient air sampling devices as well as for large-scale air cleaning devices. The theory is not well understood, 15 but available evidence indicates that such forces can be utilized for quantitative collection of dispersoids" in air over a very large size spectrum. Heretofore, electrostatic precipitators have been used primarily for quantitative collection of dust or aerosol material to determine total concentration or activity measurements rather than for determination of size distribution. Although some methods for determining sizes from deposits thus obtained have been used, these in general may alter the nature of the dispersoid or simply may not employ a representative sample.

In view of the shortcomings of other procedures as listed "above and the relative lack of knowledge concerning electrostatic precipitation, it seemed desirable to investigate further the possibility of adapting this latter principle to the sampling of dilute aerosols and dusts for the determination of all of the characteristics noted above. Listed approximately in the order of their importance, the following factors are considered:

1. Quantitative collection of 211 sizes

2. Deposit of reasonable density on a small area

3. Reasonable sampling rate

4. No alteration of dispersoid during sampling

5. Minimum treatment to prevent alteration after sampling

6. Adaptability to light or electron microscopic observation, to quantitative chemical analysis, or to radioactivity assay

7. Portability

8. Capability of operation by unskilled worker.

It is implied in the above considerations that it is desirable to perform the observational and analytical methods following removal of the disperse phase from the air. Although this may not be the more ideal method, it is certainly essential for microscopic size-counting, observation of particle shape, and degree of aggregation. It is also apparent that the first five factors are aimed at obtaining a "picture" of the material in essentially the same state as it occurs in air. 
Modified Electrostatic Precipitator The conventional electrostatic precipitator which is available commercially utilizes a concentric cylindrical electrode arrangement, the aerosol deposit being obtained over a relatively large surface area, $i_{0} e_{.}$, about $200 \mathrm{~cm}^{2}$.

To achieve a deposit on a smaller area, the electrode configuration was modified to a point-to-plane arrangement. The plane or collecting electrode is a half-inch diameter stainless steel cylinder with the top surface mechanically lapped to a mirror finish. It is couplea to a 1 rpm synchronous motor and rotated during sampling. The point or charging electrode is a chromium plated, high-speed rotary file containing eight symmetrically spaced sharp points on a circle approximately $1 / 16$ in. in diameter. This electrode does not rotate. Both electrodes are mounted in a chamber constructed of polystyrene and textolite and have a common axis. Figures $I$ and 2 are photographs showing the construction and assembly。

A direct current power supply provides the necessary high voltage. The charging electrode is maintained at about 8000 volt,s, negative with respect to the grounded collecting electrode. The corona current is adjusted by moving the collecting electrode up or down. The maximum current obtainable without arcing in the present equipment is about $75 \mu$ amp. For this current the spacing between electrodes is about $7 \mathrm{~mm}$ giving an average voltage gradient of about $11,000 \mathrm{volts} / \mathrm{cm}$.

Air flow is perpendicular to the common axis of the electrodes and enters through a rectangular opening about $12 \mathrm{~mm}$ wide and $4 \mathrm{~mm}$ high. The horizontal centerline of this opening is located about midway between the electrodes and the plane of the opening is about $11 \mathrm{~mm}$ from the electrode axis, $i_{0} e_{0}$, about $5 \mathrm{~mm}$ from the edge of the collecting electrode.

Theoretically the efficiency of such a device should be 100\% up to some. limiting flow rate above which the efficiency drops off. Preliminary experiments indicate that this limiting. flow rate for the present instrument may be about $2 \ell / \min$. These tests are continuing.

Treatment of Sample, The aerosol deposit is thus obtained on a circular surface of about $1.25 \mathrm{~cm}^{2}$. Due to the symetrical geometry of the electrodes and due to the rotation of the collecting electrode, the deposit obtained should also be symmetrical. It has been found possible to remove the deposit quantitatively from the collecting electrode surface without disturbing the spatial arrangement of the particles by encasing it in a thin plastic film. This may then be transferred to a glass microscope slide and examined in the light microscope.

To accomplish this, the surface of the collecting electrode must be clean and highly polished before sampling. After a sample is taken, the electrode is removed from the precipitator and a small drop of $2 \%$ nitrocellulose in redistilled amyl acetate is placed at the center. Tilting and rotating the electrode spreads the solution over the entire surface which is then left to dry, usually for about 15 minutes. 


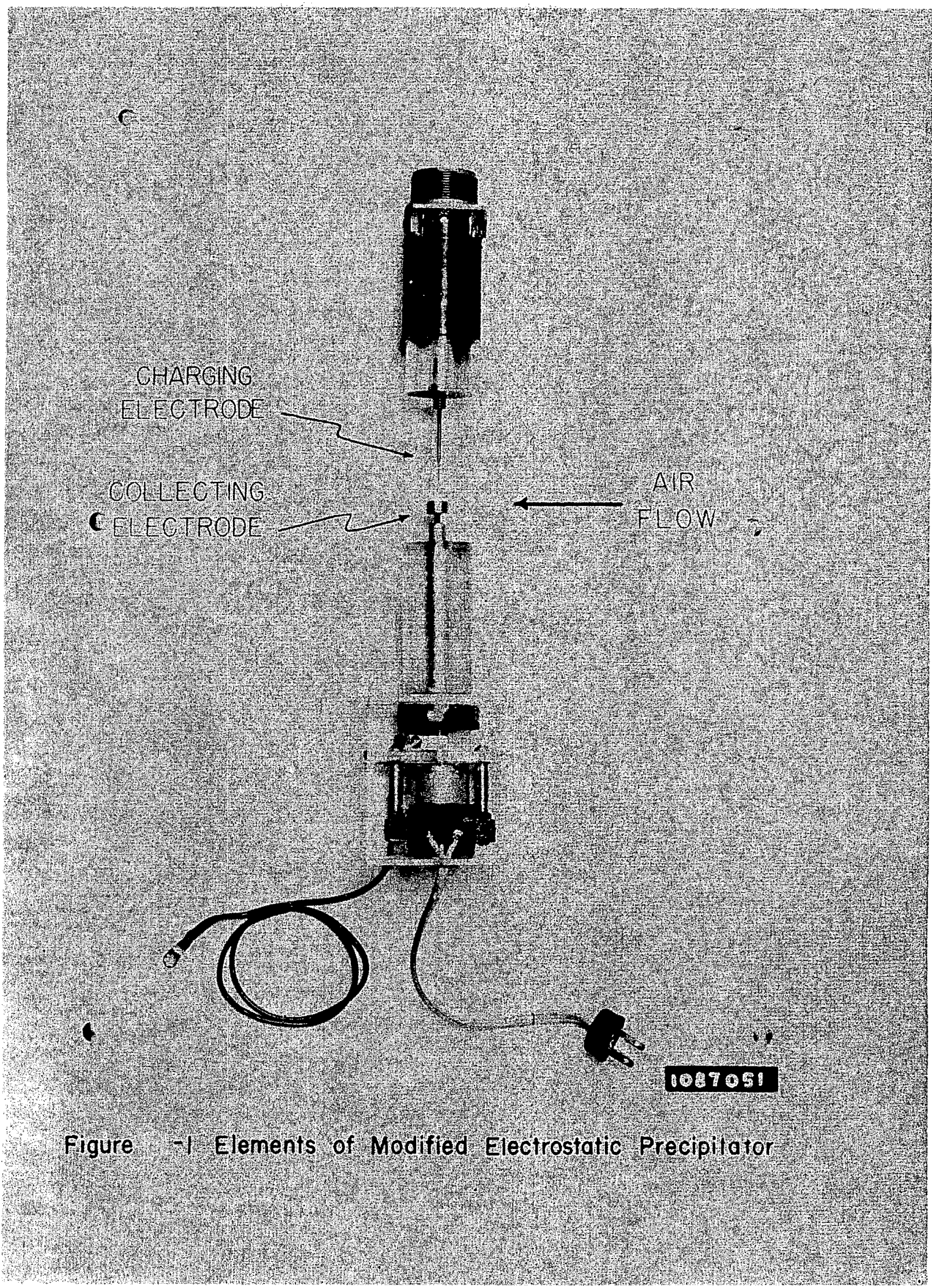




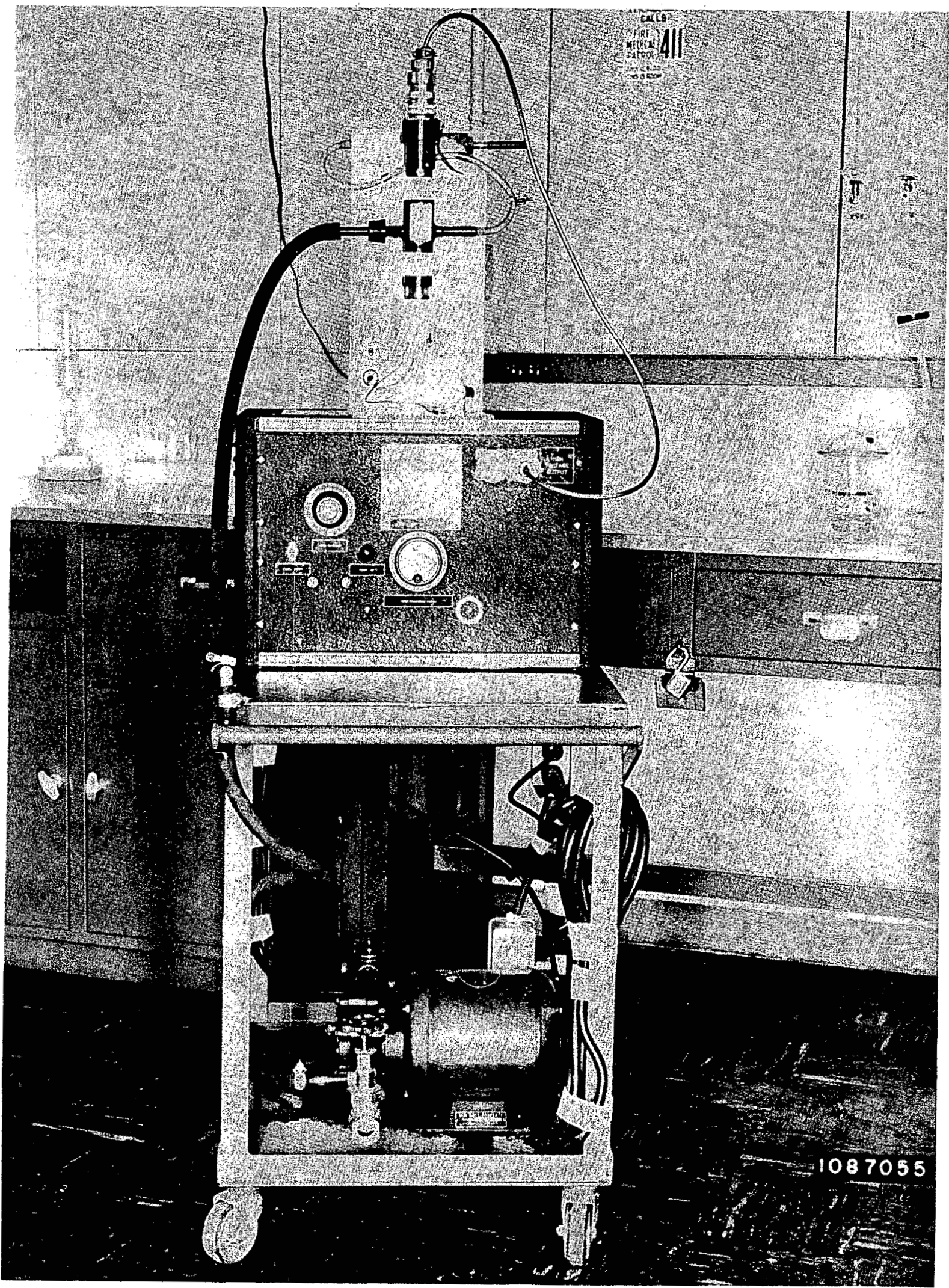

Figure-2 Complete Sampling Assembly 
Following this procedure the film may be removed by floating it of onto a water surface, taking advantage of the surface tension of water (Figure 3 ). Sometimes, soaking in water for 10 to 15 minutes is necessary before the film can be floated off. This thin circular film may then be placed on a glass microscope slide and covered with a cover glass.

Films prepared in this way appear to be of the order of 1 to 10 microns in thickness and have about the same refractive index as glass microscope slides.

Analysis of Sample with Light Microscope. The microscope employed for this work is a Spencer research type with a binocular eyepiece and a mechanical stage. The latter is provided with a vernier scale in both $\mathrm{x}$ and $\mathrm{y}$ directions. For high resolution and good definition a $3-\mathrm{mm}$ oll immersion apochromatic objective is used with $15 \mathrm{x}$ compensating eyepieces, giving an over-all magnification of about $900 x$. Both the substage condenser and objective have a 1.30 numerical aperture.

Size counts are made with an eyepiece scale $5 \mathrm{~mm}$ long with 50 divisions. This is calibrated against a stage micrometer for the particular combination of eyepieces and objective used. For the combination noted above, one division $=1.77$ microns. The method consists of placing the scale in a horizontal position and counting all particles found in the area bounded by the center and outer edge of the film and by the width of the eyepiece scale (Figure 4). The number of particles falling into each size group in 0.l-division intervals is recorded. All sizes or "diameter"s" are measured in the same horizontal direction. To facilitate the measurements, the stage is moved slowily in a horizontal direction by means of a synchronous motor acting through a variable speed changer and a belt drive.

To allow for the possibility that there is some radial distribution of size and number density, the total area seanned is divided into six intervals, each about $1 \mathrm{~mm}$ in length. In this way the size-counts may be corrected to include the entire deposit, assuming symetry about the center of the film.

Method of Interpretation of Data

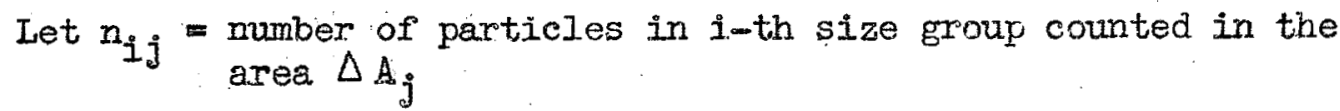

$\Delta A_{j}=$ area scanned in $j$-th section

$A_{j}=$ area of j-th section (circular or annular) and $\mathrm{n}_{i}=\begin{gathered}\text { total number of particles in } i-t h \text { size group in entire } \\ \text { deposit }\end{gathered}$

Then $n_{i}=\sum_{j=1}^{6} \frac{n_{j j} A_{j}}{\Delta \Delta_{j}}$ 


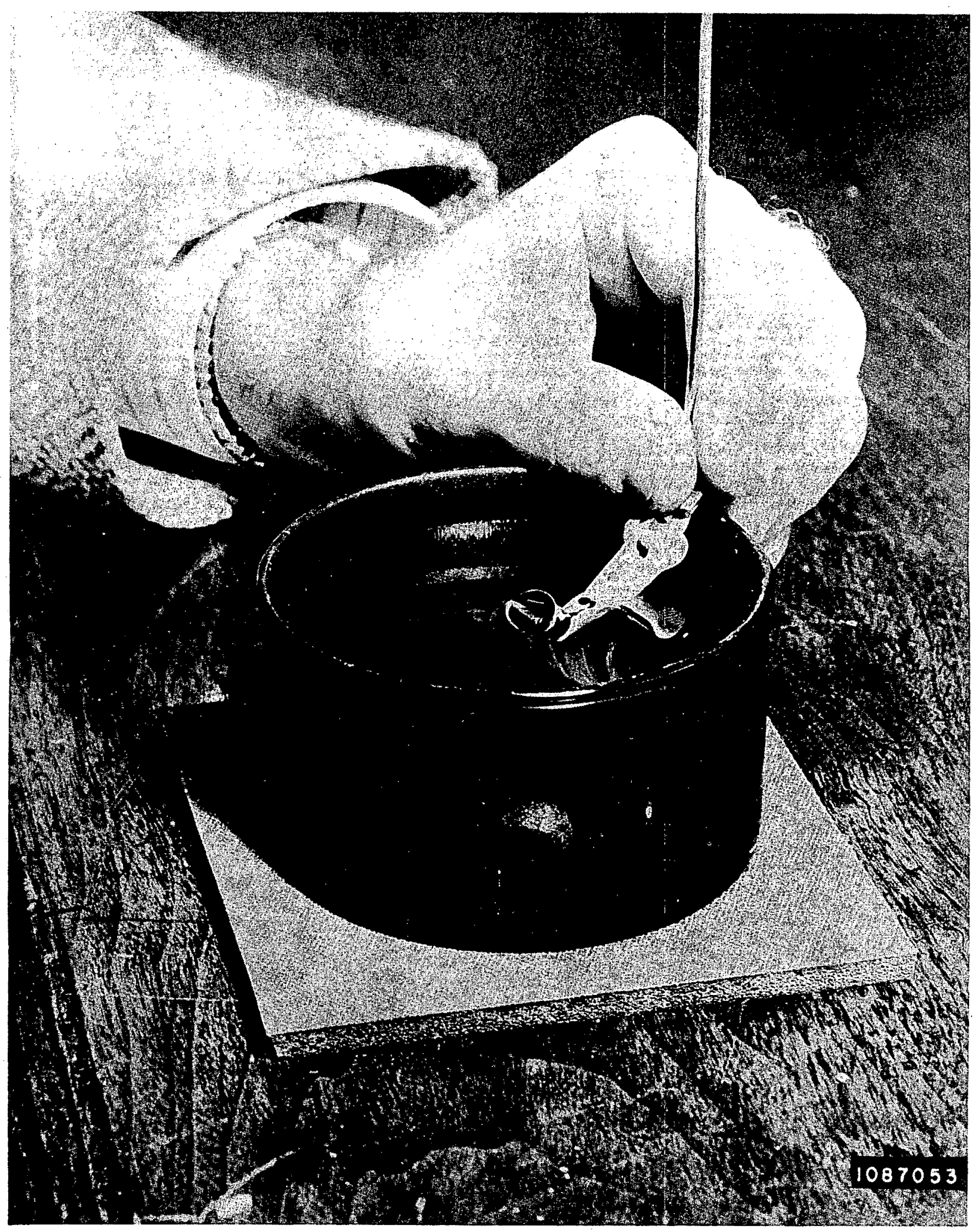

Figure -3 Removing Nitrocellulose Film from Collecting Electrode 


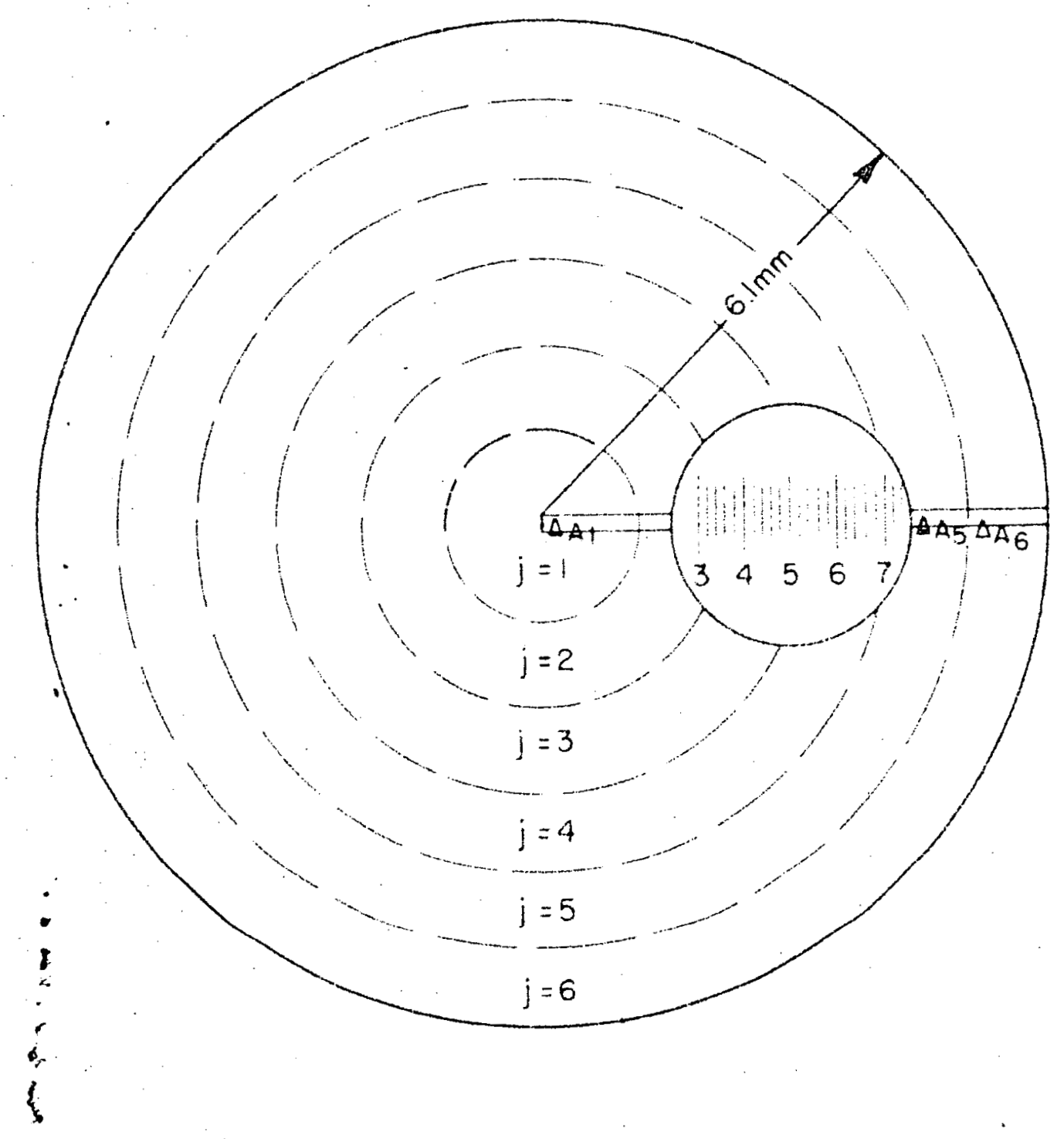

FIGURE-4 - METHOD OF SCANNING FILM 
To convert to a mass basis, let

$$
\begin{aligned}
& M_{i}=\text { total mass of particles in i-th size group in entire deposit } \\
& P=\text { density of particulate material } \\
& \phi=\text { volume shape factor }\left(\frac{\pi}{6}\right. \text { for spheres) }
\end{aligned}
$$

Then $M_{i}=a_{a i}^{3} \sum_{j=1}^{6} \frac{n_{i j} A_{j} \phi_{p}}{\Delta A_{j}}$

Where $d_{a i}=$ average size of icuth group.

Assuming $\phi$ and $\rho$ are constant or may be represented by average values,

(2) becomes

$$
M_{i}=\phi_{\rho} n_{i} d_{a i}^{3}
$$

The total mass of the deposit is then given by

$$
M=\sum_{i=1}^{m} \phi_{\rho} n_{i} d_{a j}^{3}
$$

Where $m=$ total number of size groups .

Again, if $\phi$ and $\rho$ are independent of $i$ or may have average values,

$$
M=\phi \rho \sum_{i=1}^{m} n_{i} d_{a i}^{3}
$$

In general, the particulate material would be known or identifiable, and therefore, the density would be known. The average volume shape factor would not generally be known, although by visual inspection it could readily be determined if the departure from the value for spheres would be large. For most irregularly shaped materials $\phi$ would be expected to be somewhat less than $\pi / 6_{0}$ For aerosols which show a high degree of flocculation, the volume shape factor would be highly variable due to the "porous" particle structure.

In the present case it should be possible to evaluate $\phi$ in a concentration range where a chemical analysis can be carried out on a given sample after it has been size-counted. There would be no a priori reason to suppose that the extrapolation to lower concentrations would be invalid as long as the degree of flocculation is small in both cases.

In any event evaluation of $\phi$ would be unnecessary for the determination of the mass-size distribution if $\phi$ is assumed independent of i. Such a 
distribution curve could be readily obtained by plotting the upper size limit of each group against the ratio

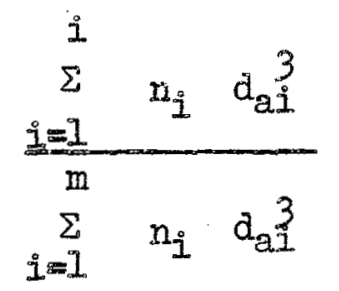

and the mass median diameter is given by the value of 0.50 for this ratio. It is apparent that any other type of statistical diameter desired could be determined from the same basic data. The method also is independent of the assumption of any particular kind of size distribution but allows the determination of the latter.

Other Analytical Methods, This procedure has not yet been adapted directly to electron microscopy. It is probable that the films thus far prepared are too thick for use in the electron microscope. Thinner films have been prepared by redissolving in amyl acetate and redispersing according to customary techniques. Inasmuch as this process would normally separate most loosely bound aggregates, it may not always be possible to secure a representative sample in this manner.

The adaptation of the method to chemical and radioactivity assay is apparent. For the latter, the size of the electrode and deposit allow direct counting procedures without further disturbing the material. The lack of any interfering medium for absorption of alpha radiation would seem particularly desirable. Quantitative removal of the deposit in an ashless nitrocellulose film also makes the problem of chemical analysis relatively simple. In these respects it is recognized that the device would be of only limited utility if the maximum usable flow rate is only $2.0 \mathrm{k} / \mathrm{min}$, since this might not be sufficiently high for concentration determinations of extremely dilute aerosols. It is hoped that this limit can be raised.

Results on Evaluation of Modified Electrostatic Precipitator Efficiency It is difficult to conceive a method for the measurenent of efficiency of any aerosol sampling device on an absolute basig for reasons enumerated above. Considerable experimental and theoretical justification exists, however, for postulating the idea that the efficiency of an electrostatic precipitator can be made as nearly perfect as one may desire. It is, therefore, not unreasonable to evaluate the efficiency of the modified electrostatic precipitator on a relative basis, $i_{0} e_{0}$, with two such devices in series.

In such a determination background "dust" on the electrode prior to deposition, plus that remaining on the surface of the microscope slide even after thorough cleaning, becomes a significant factor. Although no such measurements have yet been made, it is improbable that this background count can be reduced mach below about 25 particles/m2. Without allrowing for this background count, one efficiency test has given $99.95 \%$ on a mass basis for sampling ordinary laboratory air at a rate of $2.0 \mathrm{k} / \mathrm{min}$. Investigation of the

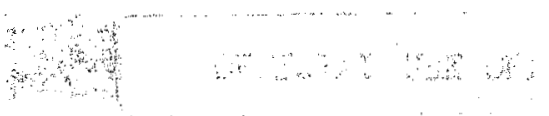


apparently important effect of humidity discussed belowg has delayed getting more of this type of data temporarily.

Characteristics of Deposit As indicated above, the air inlet was positioned to favor deposition on the top surface of the collecting electrode. This has been qualitatively checked by sampling with two precipitators in parallel. The surface concentration of material from the top surface of one electrode was found to be several orders of magnitude greater than the concentration of material removed from the side surface of the other electrode。

Figure 5 shows the results of measurements on a sample, giving the radial distribution of number density along four radii taken $90^{\circ}$ apart. The points represent the concentration of particles of all sizes in number per square millimeter taken at average radial distances from the center, $i$ oe.

$$
\frac{n_{j}}{\Delta A_{j}} \text { vs } \frac{r_{j}-r_{j-1}}{2}
$$

On a number basis this represents the "worst". degree of symmetry obtainable. In the usual case nearly one half of the total number of all particles is in the smallest size group, and experience has shown that the number in this group seems to vary over rather wide limits. On a mass basis, therefore, the four curves would be very nearly identical. It is obvious that more evidence is needed to confirm this point.

Number counts of several similar sampies along only one radius show that the radial distribution is not always of the same type as shown in Figure 5. Figure 6 shows the results of a complete radial size-count on a sample of metallic dust. The radial distribution of number density (Figure 6a) is markedly different from that observed in Figure 5. When modified to give mass density, the distribution is somewhat flatter (Figure 6b). Of particular interest is the radial distribution of size. Figure $6 c$ shows some evidence that larger particles are deposited closer to the center of the collecting electrode, giving rise to a larger mass median diameter in the central section, $\triangle A_{I^{\circ}}$

Effect of Humidity of Sampled Air At irregular intervals it has appeared that the precipitator was not operating at the normal high collection efficiency. Furthermore, a yellowish corrosive deposit would appear on the collecting electrodes at the same time. After considerable experimentation it was found that the humidity of the air is a very important factor. Preliminary results indicate that operation of the device is generally satisfactory when the relative humidity at room temperature is over $30 \%$ but is not satisfactory at lower humidities.

Size Distribution of KAPL Shop Dust

Extending over the past two years there has been considerable interest in determining the size spectrum for uranium and beryllium dusts in KAPL shops. 


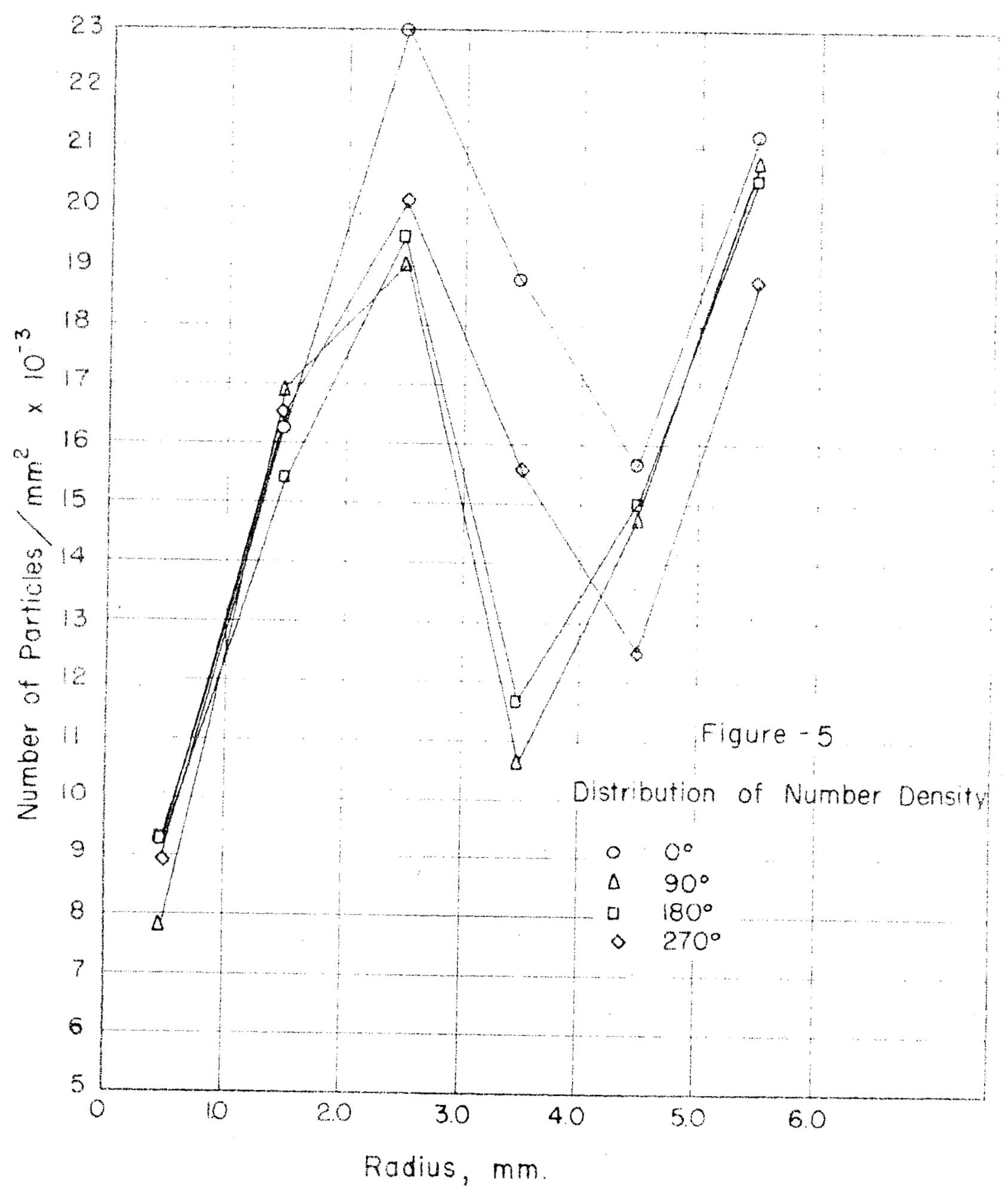




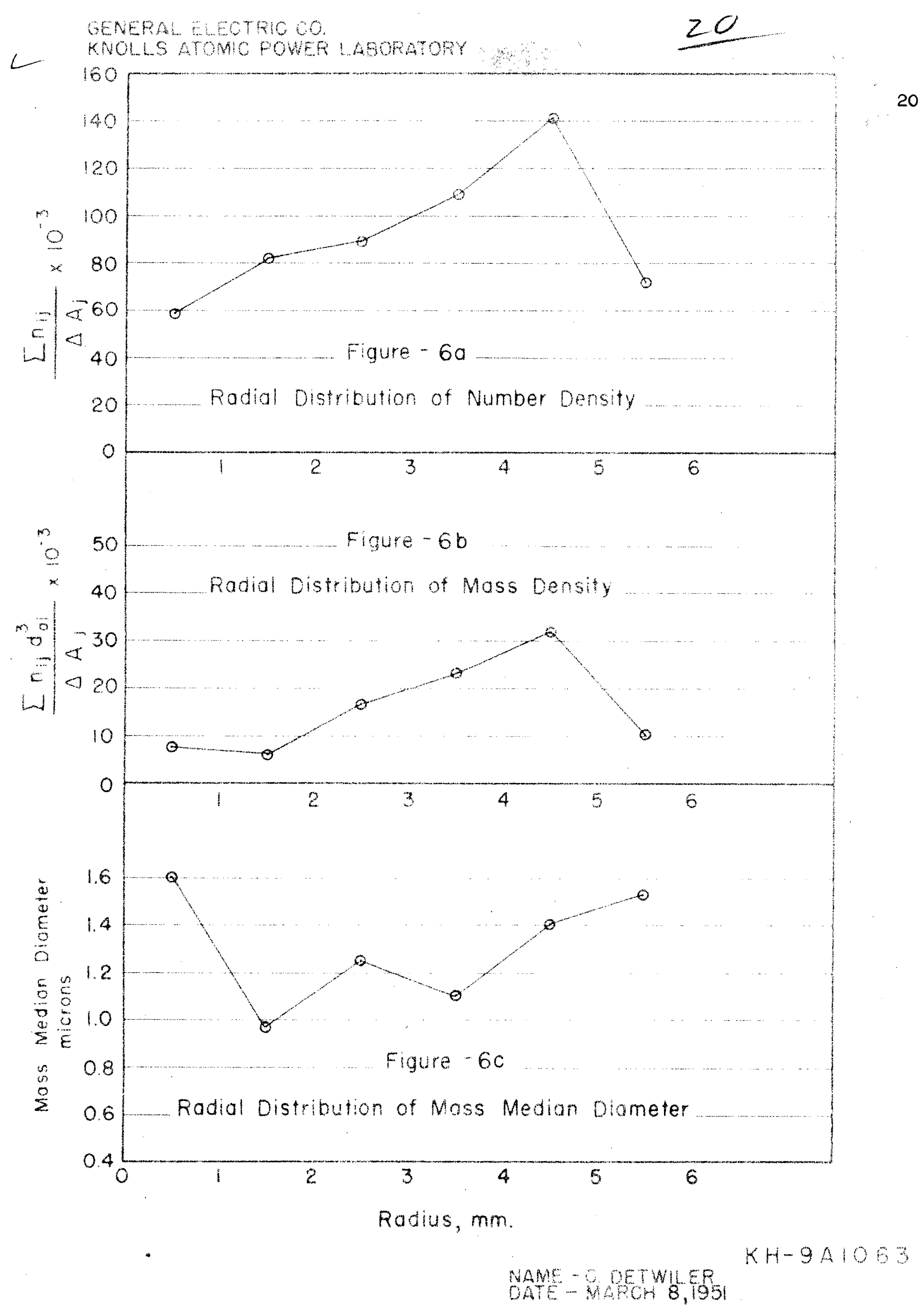


The earliest attempt utilized a tranquil settling procedure and electron microscopy. 16 Although these results were inconclusive, they indicated mean diameters of about $0.25 \mu$ for beryllium and about $1 \mu$ for uranium.

Conventional Mine Safety Appliance Company electrostatic precipitators were next used to deposit directly on electron microscope specimen screens placed at various positions on the aluminum foil liner. A number of electron micrographs of material obtained in this manner resulted in the conclusions that the mean sizes were probably of the order of 0.25 to 0.5 microns. Inasmuch as there was considerable lack of assurance of obtaining a representative sample by this method, it was abandoned.

A modified cascade impactor has been used in this work also, but in general the concentrations, particularly of beryllium, are too low to take advantage of the prior calibration. 5 A four-hour sample taken at the standard flow rate of $14 \mathrm{l} / \mathrm{min}$ at a distance of $3 \mathrm{ft}$ from a beryllium turning operation yielded less than $0.05 \mu \mathrm{g}$ on each of the five stages, which is the limit of sensitivity of the spectrographic procedure used here. It is interesting to note that one could obtain the same result with about $2.5 \times 10^{6}$ beryllium particles of diameter $1.0 \mu$ evenly distributed over the five stages.

It therefore appeared that a more sensitive procedure would be desirable, and this is one reason the instrumentation project described above was undertaken.

Some size data have already been obtained with the modified electrostatic precipitator. These are of a preliminary nature only. No attempt has yet been made to identify specific materials in the light microscope, but transparent nonmetallic materials have been excluded from the size counts. All of these data were taken before the collecting electrode was made to rotate. The deposits were, therefore, redispersed, and there is little Ilkelihood that any large flocs will remain unbroken. The data are shown below.

\section{Sample Description}

Fresh Alr to Machine Shop

S-4, Be turning, at 8 in.

S-5, Shop Gen'I Atmosphere

S-5, Shop Gen'1 Atmosphere

S-2, Shop Gen'1 Atmosphere

S-11, Shop Gen'I Atmosphere
Mass Median
Diameter

$0.8 \mu$

$1.6 \mu$

$1.6 \mu$

$2.0 \mu$

$1.8 \mu$

$2.0 \mu$
Largest

Diameter

$1.2 \mu$

$3.0 \mu$

$4.0 \mu$

$4.0 \%$

$3.0 \mu$

$4.0 \mu$
Remarks

From Electron Micrograph

$$
\begin{array}{r}
\text { Lt. Mic., } 3 \text { mobj. } \\
3 \text { obj. } \\
8 \text { obj. } \\
3 \text { objo } \\
3 \text { mm obj。 }
\end{array}
$$

The following conclusions might be drawn from the above data: (a) machine shop operations not only increase the concentration of dust in the shop but

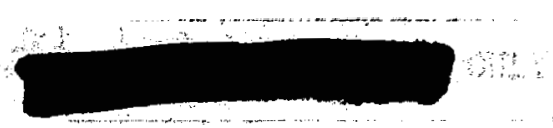


also the particle size; (b) virtually all of the dust is in the size range where alveolar retention is appreciable; 8 (c) the increase in resolution obtainable by shifting from an 8-mm objective with a dry mount to 3-mm oil immersion changes the mass median diameter by a significant amount. Considerably more data are needed to confirm the conclusions (a) and (b). This is to be obtained with the modified electrostatic precipitator in the near future.

An effort will be made to express analytically the size distribution data obtained in this study. Most of the data obtained thus far are adequately represented by straight lines on logarithmic-probability grids. Roller's equations 17 , If are to be tried, however, since they may conveniently be used to express bimodal distribution and regression, two factors which may be significant in this type of study。

Aerosol Filter Tests

One of the simplest means of determing the concentration of a toxic or radioactive aerosol involves sampling through porous filter media. Knowledge of the filtering efficiency for aerosols of such media is of prime importance for accuracy in such determinations.

Theoretical considerations 19 show that the efficiency or, conversely, the penetration of a filter should depend on the particle size distribution and density of the particulate material and the fluid velocity through the filter as well as the characteristics of the filter itself. In addition, when sampling conditions yield a retained deposit whose volume is an appreciable fraction of the voids of the filter material, the efficiency may also be expected to depend on the concentration-flow rate-time product, $i_{0} e_{0}$, the total mass or volume retaine.

At the Knolls Atomic Power Laboratory two different types of filters are used for most air sampling work. For the sampling of beryllium-containing dusts, a chemical-type ashless filter paper, Whatman \#40, is employed. For aerosols bearing nonvolatile radioactive materials, an asbestos fiber-base filter paper is employed. This is manufactured by the Hollingsworth and Vose Company, East Walpole, Massachusetts, and is designated as type $\mathrm{H}-70$. Little or no data are available in the literature on the performance characteristics of these filter papers with respect to efficiency for aerosol sampling.

The principal purpose of this project is then to determine the efficiency characteristics of these two filter papers as functions of the particle size distribution and density of the aerosol and of the fluid velocity at varying values of volume retention. The secondary purpose is to establish a method for the determination of aerosol filtering efficiency which includes all important variables and which is particularly applicable to very highly efficient filters.

Scope of the Project Current plans call for the coverage of the following variables insofar as time permits:

1. Filters - Whatman $\# 40, \mathrm{H}_{0}$ \& $\mathrm{V}$ 。 H-70 



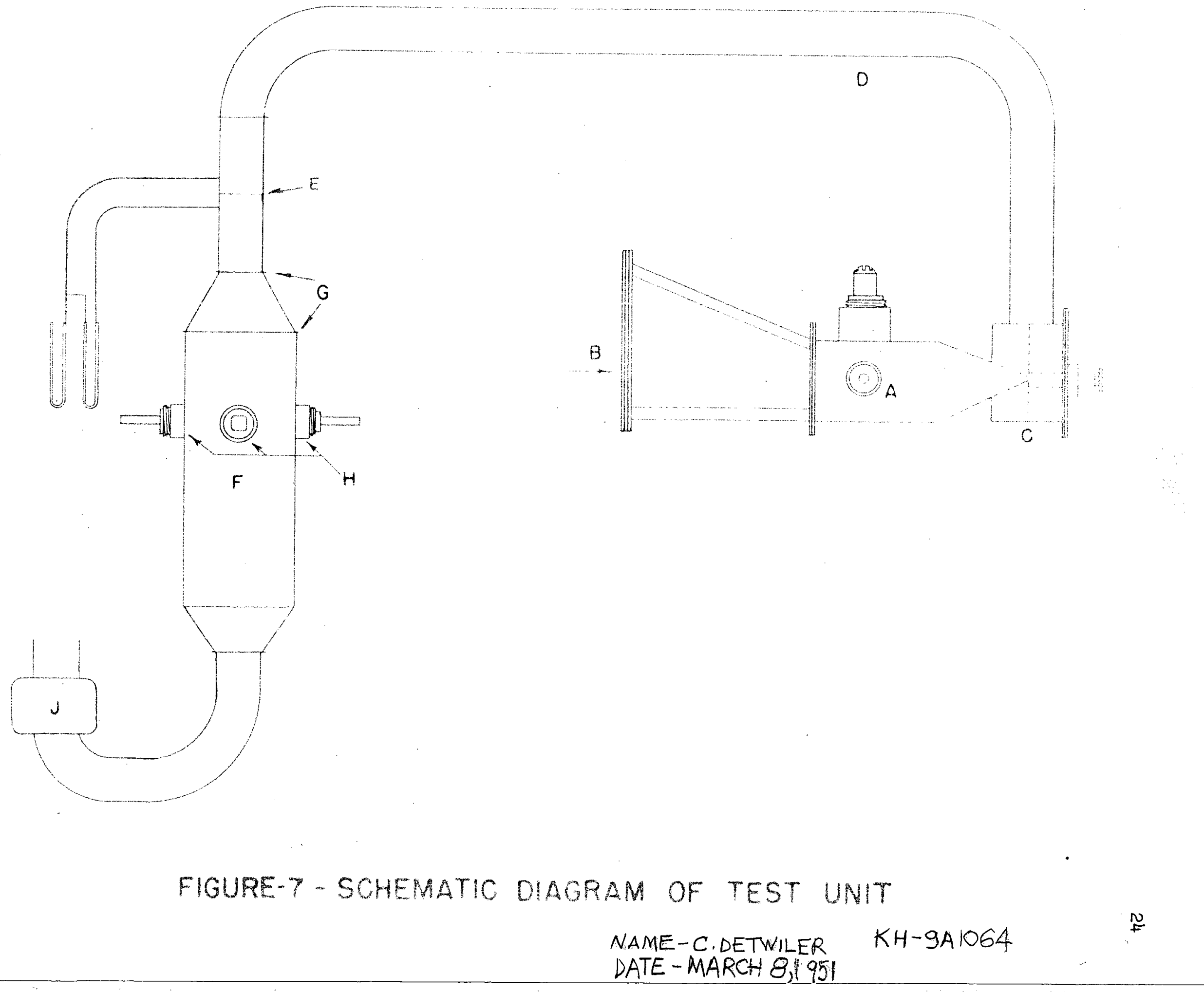




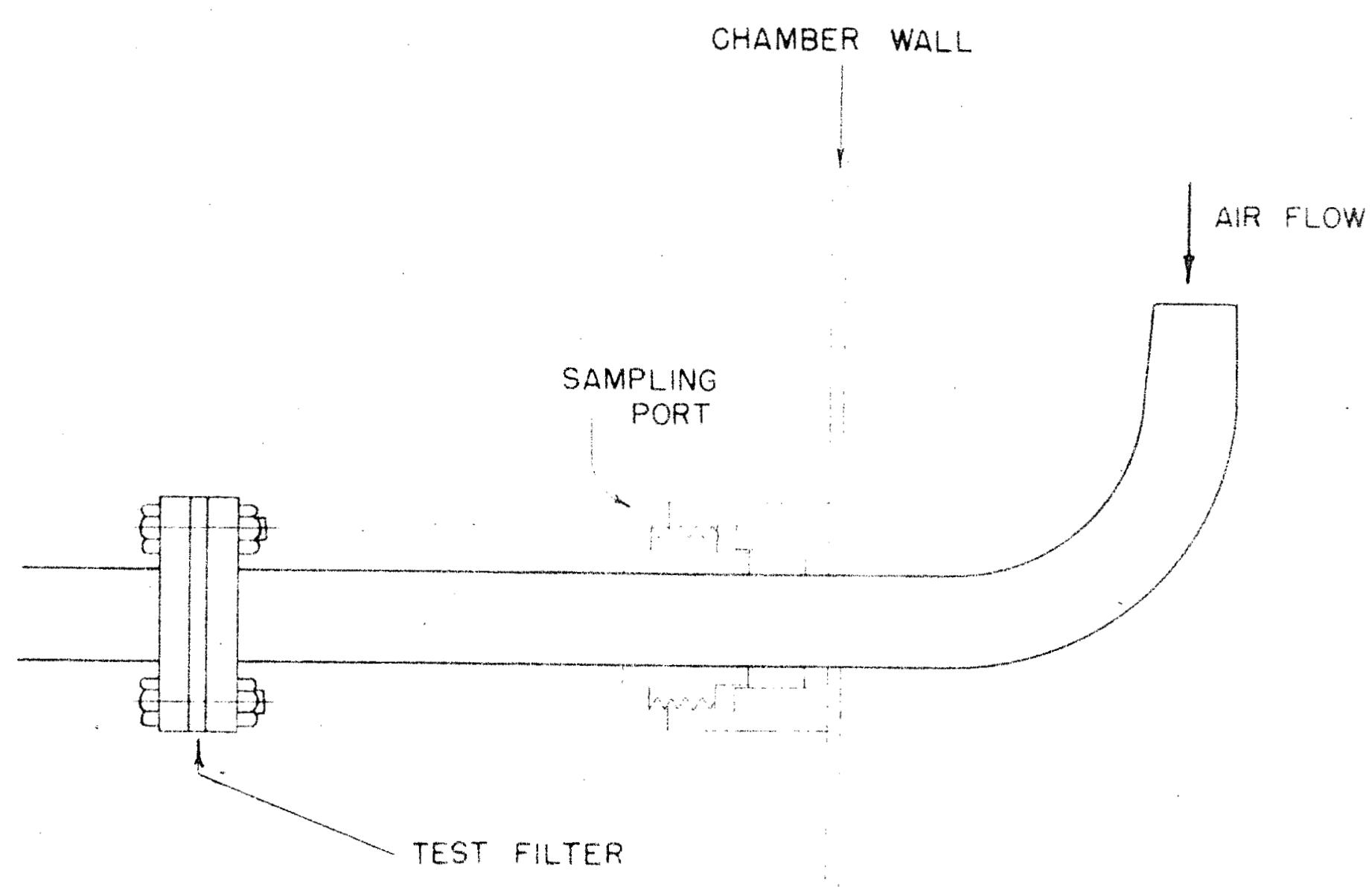

FIGURE-8 - ISOKINETIC SAMPLING HEAD 
has given a concentration of about $25 \mu \mathrm{g} / \mathrm{M}^{3}$, a mass median diameter of $1.3 \mu$, and a maximum particle diameter of 3.2 microns.

Experimental Procedure In order to determine efficiencies of filters on a mass basis, it is necessary to determine at least two of the following three quantities: (I) the mass or concentration entering the filter; (2) the mass retained by the filter; and (3) the mass or concentration leaving the filter. Where the filtration efficiency is high, considerably higher accuracy may be achieved by determing the inefficiency or penetration of the filter directly. This involves determination of the third of the above items, in addition to either the first or second. To achieve this, one electrostatic precipitator is connected directly to a sampling port on the chamber without any filter between the flanges and a second is connected just downstream from the test filter, which is located in a second sampling tube, Each precipitator is followed by a calibrated capillary-type flowmeter for individual adjustment of sampling flow rate. Both are connected to an auxiliary air pump for drawing the air samples, since the chamber is under a slight vacuum. Variation of the linear velocity through the filters is achieved primarily by varying the crosssectional area open to air flow, since the chamber and sampling volumetric flow rates are linearly dependent under the condition of isokinetic sampling. The maximum sampling rate and, consequently, the maximum chamber flow rate is fixed by the upper limit for $100 \%$ efficiency operation of the electrostatic precipitators.

Following adjustment of the facilities for passing the test aerosol through the chamber, the predetermined sampling fllow rates may be set and the precipitators put into operation. It is necessary to eliminate or to correct the results for any time lags that may alter the relative amounts of material found on the electrode deposits or in the filter. Such discrepancies would be diminished by lengthening the total time for any run. At the termination of a mun the electrode deposits may be removed as described previously.

For concentrations initially in the range of 0.1 to $50 \mu \mathrm{g} / \mathrm{M}^{3}$, exit concentrations might not always be sufficiently high for accurate chemical analysis. For this reason it was desirable to develop a procedure which would give considerably better sensitivity and accuracy for the mass determination in this low range. Inasmuch as size data were also required, it seemed possible to combine these two types of analyses into one, using only a high-power microscope as the ultimate analytical tool.

For the size-counting procedure discussed previously, if the volume shape factor $\phi$ is the same for the aerosol entering and leaving a filter, knowledge of this factor is unnecessary for the calculation of the ratio of masses of deposit on the two collecting electrodes placed before and after a filter. Denoting a sample taken with the modified electrostatic precipitator prior to a filter by subscript A and a sample taken after the filter by subscript $B$, then the efficiency $E$ on a mass basis is given by 


$$
I-E=\frac{M_{B}}{\Pi_{A}}=\frac{\left[\begin{array}{ccc}
m & & \\
i=I & n_{i}^{3} & a_{i}^{3}
\end{array}\right]_{B}}{\left[\begin{array}{rrr}
m & & \\
\Sigma & n_{i} & d_{a i}^{3}
\end{array}\right]_{A}}
$$

Further, $E$ is determined automatically as a function of particle size in the range covered by the $m$ size groups, $i_{0} e_{0}$, for any given size:

$$
1-E_{i}=\frac{\left[\begin{array}{ll}
n_{i} & d_{a i}^{3}
\end{array}\right]_{B}}{\left[\begin{array}{ll}
n_{i} & d_{a i}^{3}
\end{array}\right]_{A}}
$$

Thus, each individual run should give not only one efficiency figure applicable to the given filter and flow rate, but also a series of m efficiencies for each size group down to the limit of resolution of the light microscope, i.e., about 0.25 for the combination noted above。

Recognizing that microscopic size-counting is a tedious and time-consuming operation, it is intended that data from this source will be supplemented whenever possible with quantitative chemical analyses. This will be possible when penetrations are sufficientiy high to bring the exit concentration into the "sensitive" range of the chemical analysis and where the size-distribution data can be reliably extrapolated from previous measurements.

In the case of the Whatman-type paper, it will be possible to carry out material balance calculations by analyzing for the material retained on the paper. At the low concentration levels of interest here, this will not be practicable with the asbestos paper.

These chemical analyses will be carried out by spectrophotometric or colorimetric methods by the Analytical Section of the KAPL Chemistry Division.

Interpretation of Data Possible methods of correlation of the efficiency data obtained here have not yet been worked out. It is hoped that the experimental results can be explained on the basis of diffusion and impaction mechanisms, making use of Langmuir's theory of filtration. -9 , in effort will also be made to extrapolate the results to smaller particle siz: $=$ on the basis of this theory. 
TRITIUM DETERMINATION IN AQUEOUS SAMPLES

The radioactive isotope of hydrogen known as tritium is a very weak beta-emitter (18.5 kev max energy) with a relatively long half-life (12 years). Such a low energy precludes the possibility of mounting tritium-bearing samples as liquids or solids followed by counting with a customary thin-wall GeigerMueller tube because of absorption in the sample and the tube wall. This fact makes it necessary and desirable to utilize a gaseous sample and incorporate it in an ion chamber or counter.

\section{Procedure}

For the analysis of tritium in urine or other aqueous samples, the scheme utilized by White et al 20 has been adapted for use in this laboratory. Tritiated water is reacted with finely divided aluminum carbide in an evacuated sample tube A (Figure 9) maintained at $70^{\circ} \mathrm{C}$ by means of a surrounding water bath. The reaction is allowed to proceed for $30 \mathrm{~min}$ and is followed by partial purification of the tritiated methane at dry ice temperature for 15 minutes. This gas is then pumped into the specially constructed counter tube $B$ by means of the Toepler pump $C$ to a pressure of 0.5 at. as measured by the manometer. Construction of the counter tube is shown in Figure 10. The counter tube is connected directly into the preamplifier of an Instrument Development Laboratories methane proportional alpha counter, Model 117, which otherwise is unchanged.

\section{Results}

Figures 11 and 12 show the counting-rate characteristics as functions of the voltage and gain. The background counting rate, utilizing distilled water to prepare methane or using tank methane, is about $55 \mathrm{c} / \mathrm{min}$ corresponding to about $2 \mu \mathrm{c} / \mathrm{l}$ in the sample. The tube has also been found to be gamma sensitive. Inasmuch as no quenching circuit is employed, the above facts seem to indicate that operation of the tube is in the region of limited proportionality, i.e., the gas amplification is very high, but the discharge does not spread along the wire.

The method has been "calibrated" against five urine samples obtained from two other sites, and an average deviation of $\pm 13 \%$ was found for these samples which ranged from 7 to $70 \mu \mathrm{c} / \boldsymbol{R}$. An attempt is being made to obtain a more reliable calibrating standard.

The anticipated counting rate based on the stoichiometric reaction and equal distribution of the isotope between the reaction products is about five times that actually found. This discrepancy is undergoing further investigation.

Contamination of the counter tube by samples of relatively high activity has been obserred. Flushing the tube several times with tank methane is usually sufficient to bring the background counting rate back to normal. 


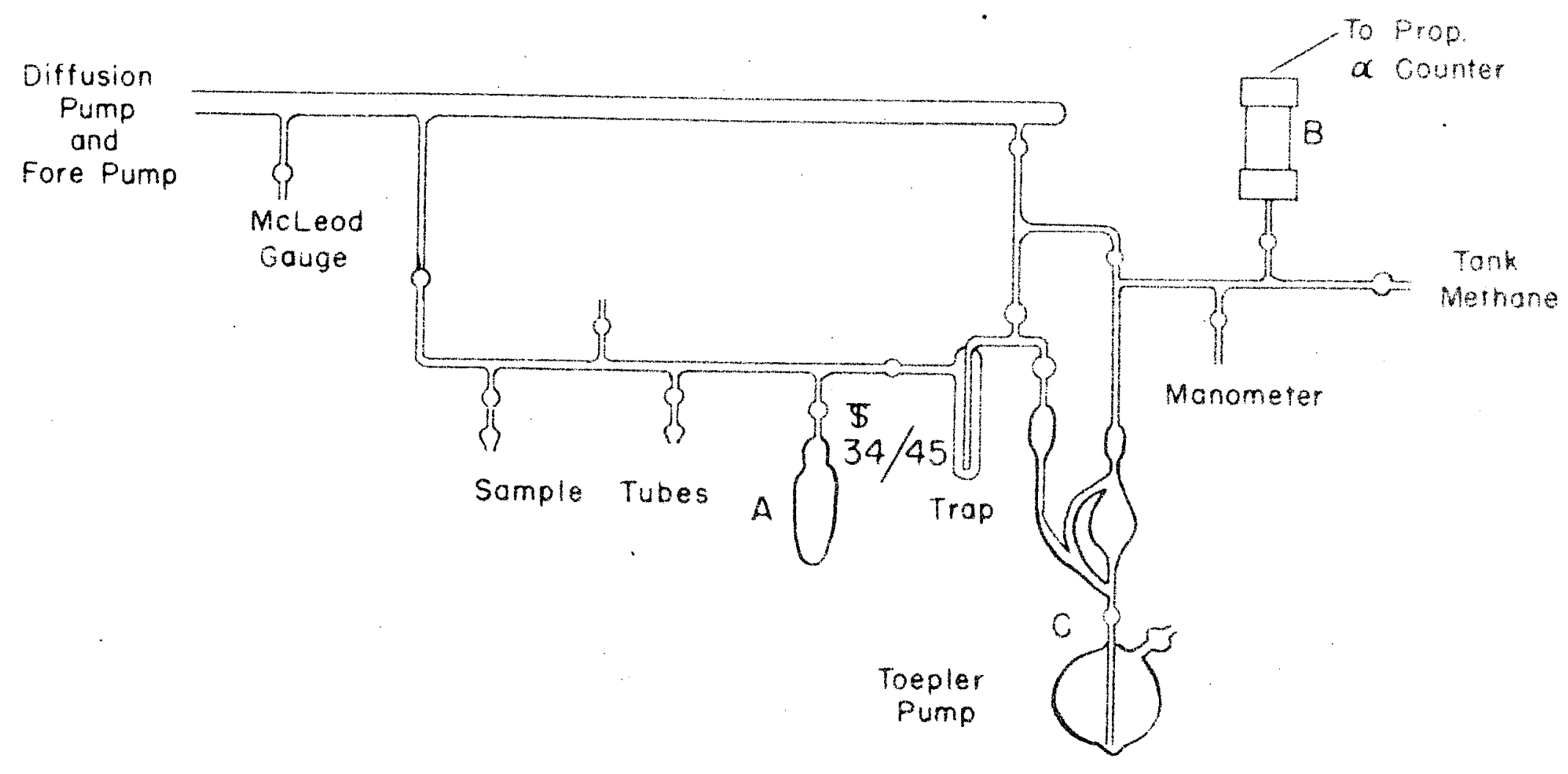

Figure - 9 Apparatus for Determination of Tritium in Water 


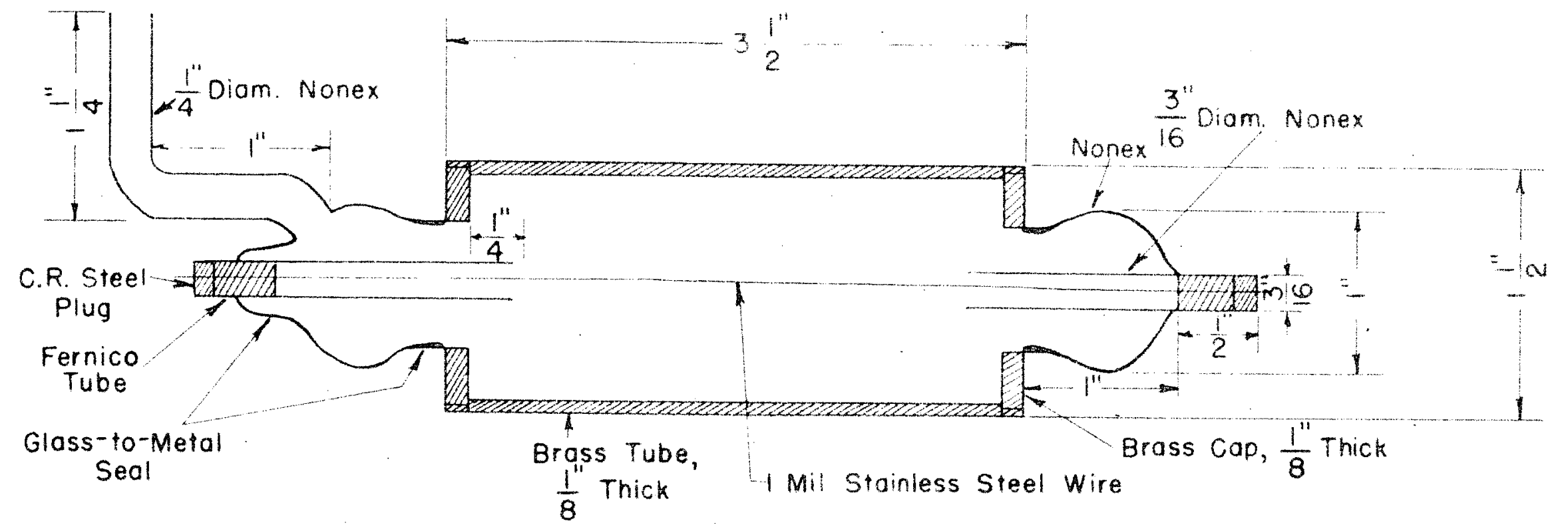

$\frac{1^{\prime \prime}}{8}$ Thick

Brass Cap

(Soldered into Tube)

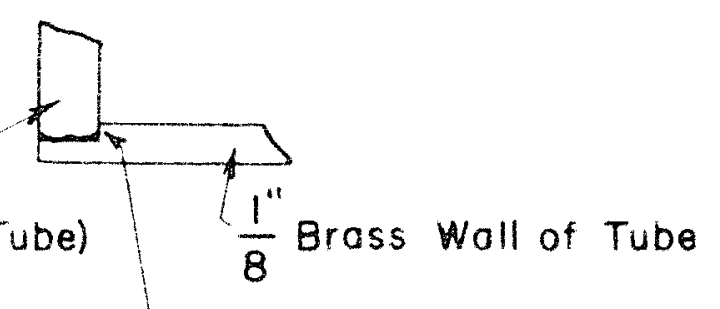

$\frac{1 "}{32}$ Lip in Brass Wall

Figure-10 Tritiated Methane Counter Tube 


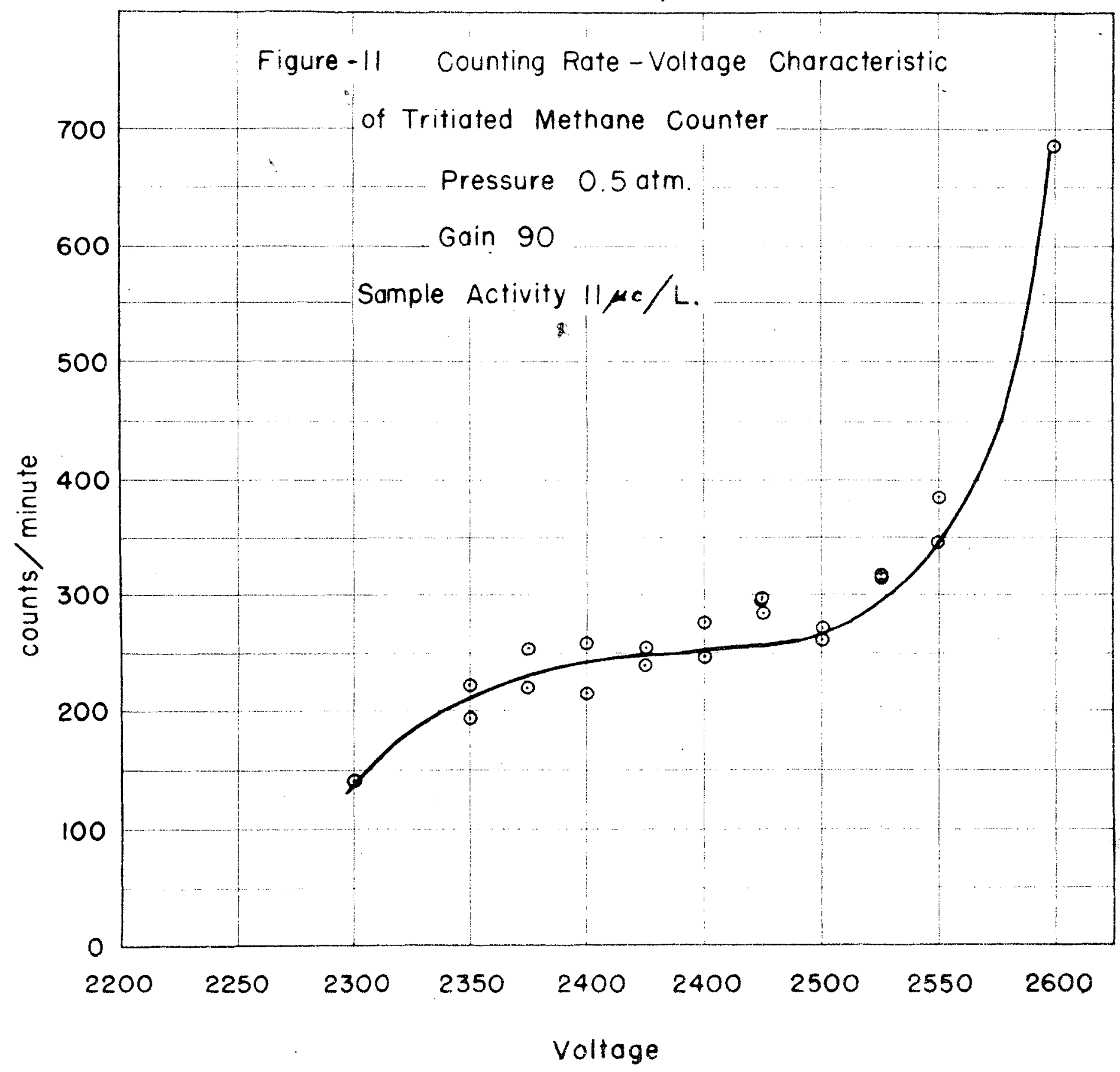

NAME-C. DETWILER KH-9A1068 


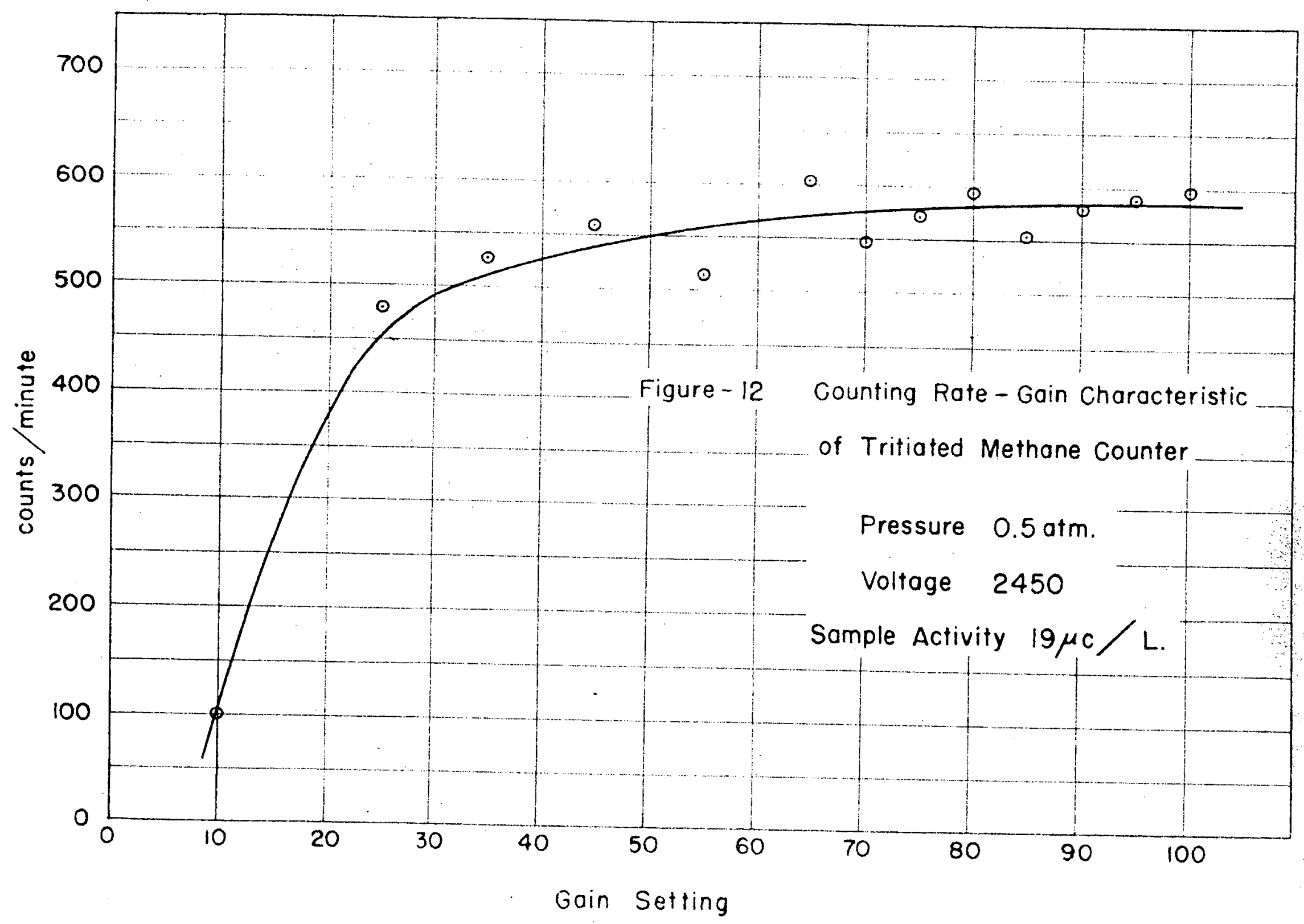


For routine urinalysis, the counter is operated at 2450 volts and one 5-minute count is taken. With the manifold system shown in Figure 9, three samples may be processed almost simultaneously, and one operator can carry - out as many as fifteen analyses in one day. 
FLUORIMETRIC ANALYSIS FOR BERYLIIUM

Introduction

The development of a satisfactory nonspectrographic analytical method for the quantitative determination of microgram quantities of beryllium has been of general interest wherever the necessity has arisen for the analysis of beryllium in air samples or biological materials.

The work outlined herein has had as its objective the development of the morin fluorimetric method and the ultimate application of this method to the determination of beryllium in air samples. The analysis utilizes the fluorescence of the beryllium-morin combination in an alkaline medium.

This project has been conducted in conjunction with N. I. Sax of the General Electric Schenectady Works Laboratory. Mr. Sax had previously been interested in the morin analysis and had partially established the conditions for its use with beryllium samples containing ostensibly no extraneous ions.

Mr. John Harley, of the ABC Now York Office, has also been investigating the applicability of the morin-berylytum analysis and has contributed optutions and ideas to the work oatitined thes

Summary and Correlustoms.

Efforts have been directed toward the development of the morin fluorimetric analysis for the determination of beryllium in air samples. A standard uranium fluorophotometer has been adapted to handle small volumes of solution and quantitative sensitivities of the order of $10^{-3} \mu \mathrm{g} / \mathrm{ml}$ have been obtained. The range of the analytical method extends from this lower level of sensitivity up to about $0.05 \mathrm{\mu g}$ of berylifum per ml. Certain cations interfere with the anglysis and mist either be separated from the sample or their effects eliminated in some other manner. Work in progress should improve the technique of removing these interfering ions.

It is indicated by the work conpleted to date that: (1) the morin analysis is superior to any of the other fluorimetric or colorimotric methods for beryllium with regard to both specificity and sensitivity; and (2) although an instrament designed for solution fluorimetry would have obvious advantages, there seems to be no reason that the typical uranium flnorophotometer cannot be utilized satisfactorily for the morin-beryllium analysis.

\section{Discussion}

As pointed out in the literature, $21-23$ morin 1 s presumed to be $3,5,7$,

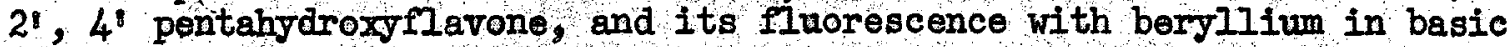
solution, when exposed to altra-violet light, is probably a function of the mumer and position of certain of the hydroxyl groups. 
The maximum fluorescence of morin seems to occur at roughly $550 \mathrm{~m} \mu$ with a continuous spectrum from $475 \mathrm{~m} \mu$ up into the infra-red. "Qualitative examination of the morin spectrum shows it to be the same with or without beryllium, the spectrum with beryllium simply being more intense.

The absorption spectrum of morin has two maxima, one ä about $263 \mathrm{~m} \mu$ and the other at $370 \mathrm{m \mu}: 24,25$ The latter peak thus coincides with the prominent $365 \mathrm{~m} \mu$ mercury line.

The analytical method discussed by Sax ${ }^{26}$ utilized a Beckman spectrophotometer with fluorescent attachment. The absorption and fluorescent spectrums of morin indicated that a uranium fluorophotometer would be suitable for this analysis. This instrument was designed primarily for the measurement of the fluorescence of solids, but its universal availability, coupled with greatly increased sensitivity over that of the spectrophotometer, made its adaptation to this analysis seem particularly attractive. The particular instrument used in this work was based on the design of the University of Rochester's Model V Fluorophotometer. 27 The necessary alterations were readily accomplished by enlarging the holes in the sample slide and employing shallow platinum dishes with a capacity of approximately $0.3 \mathrm{ml}$. There has been no difficulty with meniscus effects due to the sample position in relation to the light source which might be expected with this instrument.

Using the fluorophotometer as described, it has been possible to detect quantitatively beryllium concentrations of the order of $3 \times 10^{-4} \mathrm{\mu g}$ in the instrument or $10^{-3} \mathrm{~kg} / \mathrm{ml}$ of sample.

With berylifum solutions containing little or no contaminating ions, the morin analysis consists simply of adjusting the normality of the sample to $0.1 \mathrm{~N}$ in $\mathrm{NaOH}$, adding a predetermined amount of morin in acetone solution, adjusting the volume to conform to standard conditions, and measuring the fluorescence. Dre to slight daily variations in reagents and instrumentation, it is also necessary to run standard samples, spiked samples, and blanks along with the unknowns. The instrument readings of the standards versus their known beryllium concentrations can then be plotted and the beryllium content of the unknow samples read from the calibration curve. The relationship of beryllium concentration to fluorescence intensity is linear from the lower level of sensitivity up to about $0.05 \mu \mathrm{g}$ of beryllium per $\mathrm{ml}$, at which point self-quenching begins to distort the slope of the curve. Samples may be readily adjusted to this optimum range by dilution.

The presence of certain contaminating cations in the berryllium sample may adversely affect the analytical results in any one of three ways; namely, (I) by causing coprecipitation of the beryllium with insoluble hydroxides, (2) by contributing to the fluorescence, and (3) by quenching the fluorescence. Consequently, in the analysis of air samples it is necessary to include a separation step in the procedure prior to the addition of the morin. 
Sandell points out that iron and magnesium cause loss of beryllium due to coprecipitation upon the addition of the sodium hydroxide.28,29 Iron has not been found to be a particularly serious offender, however, when present in normal concentrations. Actually $10 \mu$ of iron per ml caused only $7 \%$ loss in beryllium in known samples. Magnesium is of greater concern since 5 Hg magnesium per $\mathrm{ml}$ caused 33\% loss. Due to the dilution factor used in these particular instances, these concentrations represent 100 times this amount in the total initial sample. Thus, ten cubic meters of machine shop air may have under certain conditions 1000 to $3000 \mu \mathrm{g}$ of iron and 200 to $500 \mathrm{\mu g}$ of magnesium.

Sandell also mentions that calcium, lithium, zinc, and scandium can cause a fluorescence with morin in basic solution. In the course of the present work, the indications have been that calcium causes the opposite effect, $i_{0} e_{0}$, decreases the fluorescence. This effect can be eliminated, however, by the addition of pyrophosphate. The contribution of zinc has been found to be negligible. Lithium and scandium have not been studied to date but would rarely be present in normal air samples:

Copper, silver, gold, manganese, and chromium are mentioned by Sandell as inhibiting the beryllium fluorescence by oxidizing the morin. For most air sample work, silver and gold can be ignored due to their rarity, and manganese has not as yet been proven detrimental. Copper and chromium, even in minute amounts, do cause substantial quenching of the fluorescence. Copper, for instance, causes $40 \%$ decrease in fluorescence when present to the extent of $1.0 \mathrm{\mu g} / \mathrm{ml}$ of sample.

Consequently, copper, chromium, magnesium, and possibly iron mast be considered as analytically detrimental and should be eliminated from the sample if dependable results are to be obtained. These metals, of course, may normally be present in a machine shop atmosphere.

Not too much is known concerning the effect of anions on the morin fluorescence. Sandell claims that silicate, phosphate, fluoride, and borate have no effect. The anions should be of little concern in this work, since they would be destroyed by the sulfuricacid's fuming, which is part of the sample preparation scheme. This preparation consists of muffling the filter paper at $500^{\circ} \mathrm{C}$ followed by sulfuric acid fuming.

The following methods have been tried for separating interfering ions or eliminating their effects. This work has been done with beryllium standards spiked with known concentrations of the Ions in question and also with'regular air samples.

Dilution Dilution is a common technique in fluorescence analyses. With the high morin sensitivity it is possible to dilute normal air samples by a factor of at least 100 in order to lower the concentration of interfering ions to a point where their effect. is negligible. Dilution alone is not dependable where these ions are present in high concentration。

Spiking In many instances it is analytically feasible to allow a certain amount of error in an analysis, provided the degree of error is known. It is possible to determine the error in this particular analysis by spiking the unknown 
with a known amount of beryllium. Again, this is not satisfactory if the factor is large。

Ammonium Hydroxide Precipitation The precipitation of beryllium with $\mathrm{NH}_{4} \mathrm{OH}$, using aluminum as an added carrier, is particularly effective in eliminating such ions as magnesium. Repeated precipitations are required to assure separation from some of the other ions such as copper and chromium.

Electrolysis Work is progressing on electrolysis at the present time. Copper, for instance, is easily eliminated in this manner, as should all the cations below ${ }^{+} 1$ in the electromotive series.

Other important factors of interest in connection with the work to date are as follows:

Fading of Fluorescence There is a pronounced fading of the morin-beryllium fluorescence when the solution is exposed to ultra-violet light after the addition of morin. This fading seems to be proportional to the intensity of the ultraviolet source. To preclude this difficulty, the fluorescence measurement must be taken immediately upon placing the sample in the fluorophotometer. Also, all samples should be read within ten minutes after the addition of the morin, since the ultra-violet component of daylight is often sufficient to cause slight fading if a longer delay is allowed.

Reproducibility There is quite a high order of reproducibility in duplicate and triplicate analyses on the same sample irrespective of the recovery obtained. Samples varying both in the concentration of beryllium and interfering ions have shown deviations from the mean, in duplicate or triplicate analyses, averaging \pm $5 \%$ for beryllium concentrations of $0.01 \mu \mathrm{g} / \mathrm{ml}$, and $\pm 10 \%$ for concentrations of $0.005 \mu \mathrm{g} / \mathrm{ml}$.

Since the elimination of interfering cations is a necessary requisite to the analysis of most air samples, further attention is being directed toward a better understanding of the effects of all common ions and also toward improved separation methods with particular emphasis on electrolysis. 


\section{LOW-LEVEL ALPHA MEASUREMENTS}

In 1948 a new method was developed in the GE. Research Laboratory by means of which it is possible to detect and identify weak alpha activities as low as 0.01 count per $\mathrm{cm}^{2}$ per hour. This signifies an improvement in sensitivity of a factor of 20 or more over standard methods presently available. An Ilford C-3 nuclear emulsion is used as a detector and the improved sensitivity is obtained primarily by employing special methods of cleaning the plates prior to exposures. Identification of specific alpha activities is rendered possible by careful range measurements. A full description of the method is to be published shortly. 30

Early in 1949 when alpha counters operated with a mach higher background than they do today, it was decided to apply this method to the study of healthphysics problems at KAPL and particularly to the study of the activity of processed urine samples. It is the purpose of this report to review briefly the findings to date and to outline what remains to be done.

One of the first discoveries made was the fact that the alpha activities of blank urine samples processed for plutonium detection varied considerably. This can be seen from a comparison of the following samples, which were deposited over a surface area of $7 \mathrm{~cm}^{2}$.

$\begin{array}{cc}\text { Sample No. } & \text { Counts per hour } \\ 3 & 4.2 \\ 6 & 0.65 \\ 8 & 5.3 \\ 11 & 0.66 \\ 12 & 0.61\end{array}$

It can be shown that these large variations are not caused by the make-up of the original samples but must be due to slight and as yet uncontrollable variations in processing. It is clear that, as long as consistently low background counts are not. obtainable the emulsion technique cannot be used, due to its outstanding sensitiy except; of course, to help determine the cause of those variations.

In the course of these investigations, the alpha activity of a variety of materials has been measured, a tabulation of which is shown as Table 1 . It is conceivable that some of the values listed might help solve the problem mentioned above. Since the sample holders were made of stainless steel exhibiting a very low contamination level, it is felt that they cannot be made responsible for these variations. On the other hand the high activity of lanthanum nitrate which is added during the processing of a urine sample is significant. This activity is sufficiently high so that it can be measured easily with an alpha counter. By this means it has been determined that as many as $18^{\circ}$ alpha counts per hour may be added with the lanthanum nitrate to each urine sample processed.

Range measurements made on lanthanum nitrate and a blank urine sample seem to indicate that: 


\section{TABLE 1}

\section{Alpha Activities of Various Materials}

Area

HP

No.

$\begin{array}{ll}1 & \text { Stainless Steel } \\ 2 & \text { Stainless Steel } \\ 5 & \text { Stainless Steel } \\ 7 & \text { Urine }\end{array}$

9 Urea

10 Urea

13 Lanthanum Nitrate

14 Lanthanum Nitrate

$25 \quad$ Platinum

34 White Gold

35 Platinum

40 Aluminum

41 Aluminum

49

50

52
Scanned No。 of

$\mathrm{cm}^{2} \quad$ Alphas

Activity

alpha $/ \mathrm{hr} / \mathrm{cm}^{2}$

Remarks

$\begin{array}{lll}2.48 & 12 & 0.05 \\ 1.05 & 17 & 0.07 \\ 5.26 & 15 & 0.012 \\ 2.92 & 13 & 0.013\end{array}$

2.50

50

0.08

2.03

56

0.12

$2.14 \quad 197 \quad 0.48$

2.20

27

0.51

3.09

\section{4}

0.07

2.08

30

0.043

3.85

114

0.088

1.50

117

0.19

1.50

126

0.21

110.011

1/2-mil, surface layer removed

1.59

5

0.01

2.16

14

0.027
Evaporated, unprocessed, not flamed

$\mathrm{CP}$

Cooked, evaporated, flamed

$1.4 \mathrm{mg}$ on standard wafer

$2.0 \mathrm{mg}$ on standard wafer
Same piece, surface untouched 
(a) The alpha activity of lanthanum nitrate is essentially due to the presence of actinium-227, including some of its daughter-product activities 。

(b) Except for a little thorium contamination, the bulk of the highlevel urine sample activity is essentially due to a carry-over of the actinium activity。

Figure 13 shows the range histogram of a fairly thin sample of lanthanum nitrate. The upper limit of this spectrum agrees well with the full ranges of Po-215 (actinium $A$ ) and $\mathrm{Po}-211$ (actinium $\mathrm{C}^{8}$ ), while the track population at shorter ranges is smeared out due to a limited amount of self-absorption within the sample. Nevertheless, the sudden increase below 26 to 27 microns can be attributed to the spectrum of Th-227 (radioactinium) and Ra-223 (actinium $X$ ) as indicated". The activity of Ac-227 proper at 21 microns could hardly be expected to show up because of its low branching ratio (1\%)。 Rn-219 (actinon) and $B I-211$ (actinium C) are also well represented by an increased population just below 33 microns. "Comparing the histogram of a blank urine sample, (Figure 14) to Figure 13 reveals the similarity of its spectrum. To be sure, the 3 tracks below 48 microns indicate some thorium contamination (Po-212 thorium $\mathrm{C}^{\prime}$ at 47.6 microns), but apart from that there is very little difference. If it can be assumed that the actinium contamination is not in equilibrium with its daughter products at the time of the first exposure, then subsequent exposures should reveal an increase in activity. This is to be checked.

Figure 14 also reveals a reasonably low track population between 20 to 22 microns. In spite of other contaminants, one could hope to detect in this region the presence of very small amounts of plutonium, the alpha range of which is 22.3 microns, as indicated. As a preliminary test, range measurements have been made on straight plutonium deposits of very low concentration. Figures I5a and $15 \mathrm{~b}$ give two such histograms; $15 \mathrm{a}$ referring to a deposit on platinum and $15 \mathrm{~b}$ referring to the same deposit but on pickled stainless steel, the standard sample holder material. Since the weight of the deposit is unmeasurable, selfabsorption should be nonexistent. Yet it is found that with platimum as a base 14\% of the tracks have deficient ranges; whereas with stainless steel as a base, $62 \%$ have been subjected to absorption. Although it is not at all clear what causes this effect, there is still a possibility of determing the minimum amounts of plutonium in urine samples that can be detected in this manner. On the basis of the above results, stainless steel would not be used as a backing for these tests.

The size and shape of samples normally prepared at KAPL is not particularly suitable for exposure to the standard 3 in. $x$ I in. plates and for speedy scanning on the microscope. A method has therefore been developed to produce one or two track linear deposits $50 \mathrm{~mm}$ long and $0.3 \mathrm{~mm}$ wide for easier scanning. The solution is fed continuously through a capillary tube to a rotating, heated, thin-walled stainless steel cylinder. After cutting and flaming, the flattened foil is exposed to the emulsion. Preliminary tests show that the efficiency of this method is close to $100 \%$ if the necessary precautions are taken. However, further confirmation of this is desirable. The linear deposit enables one in many cases to extrapolate the sample activity with reasonable accuracy from measurements made on a small 


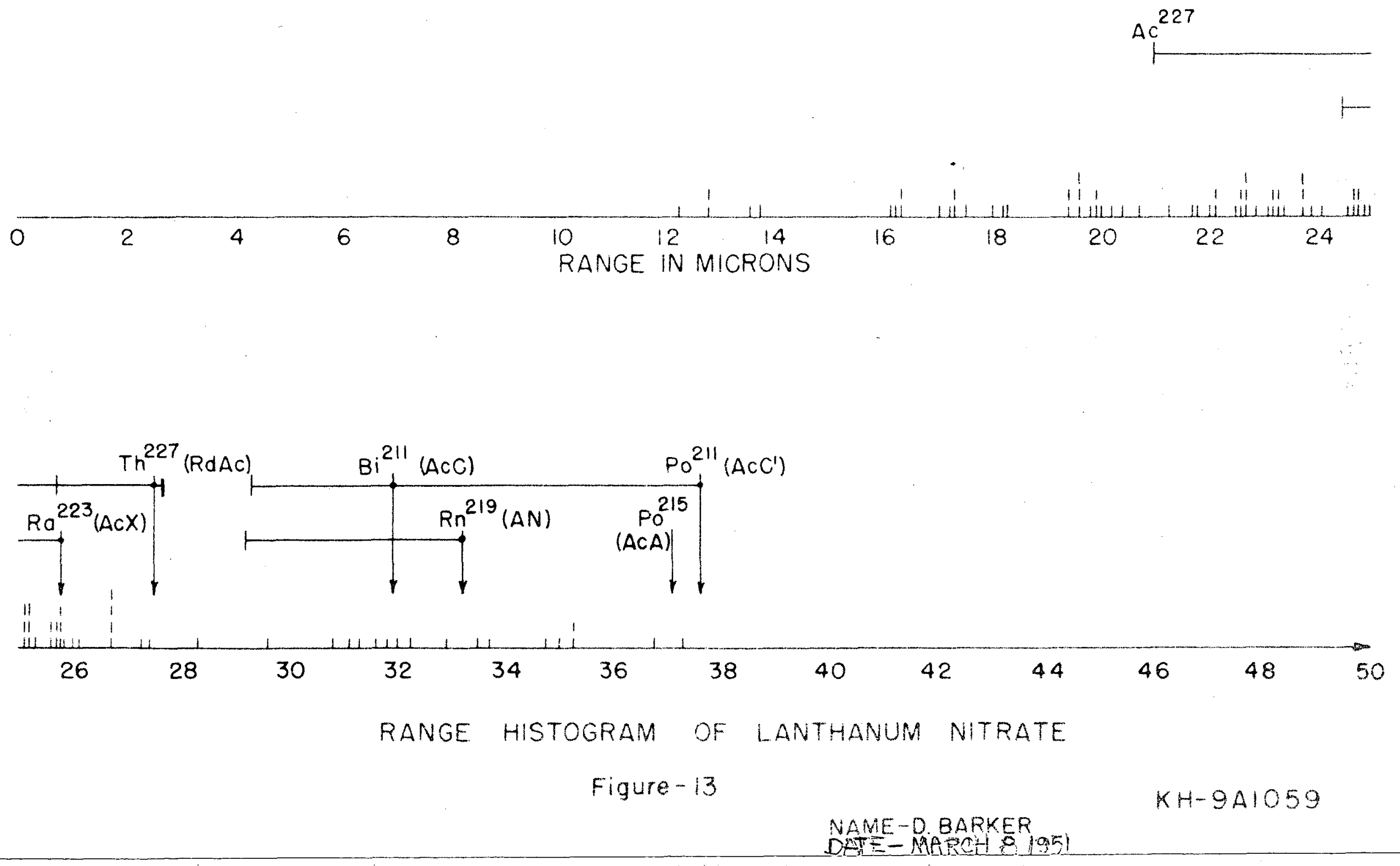




\section{H.P. 3}
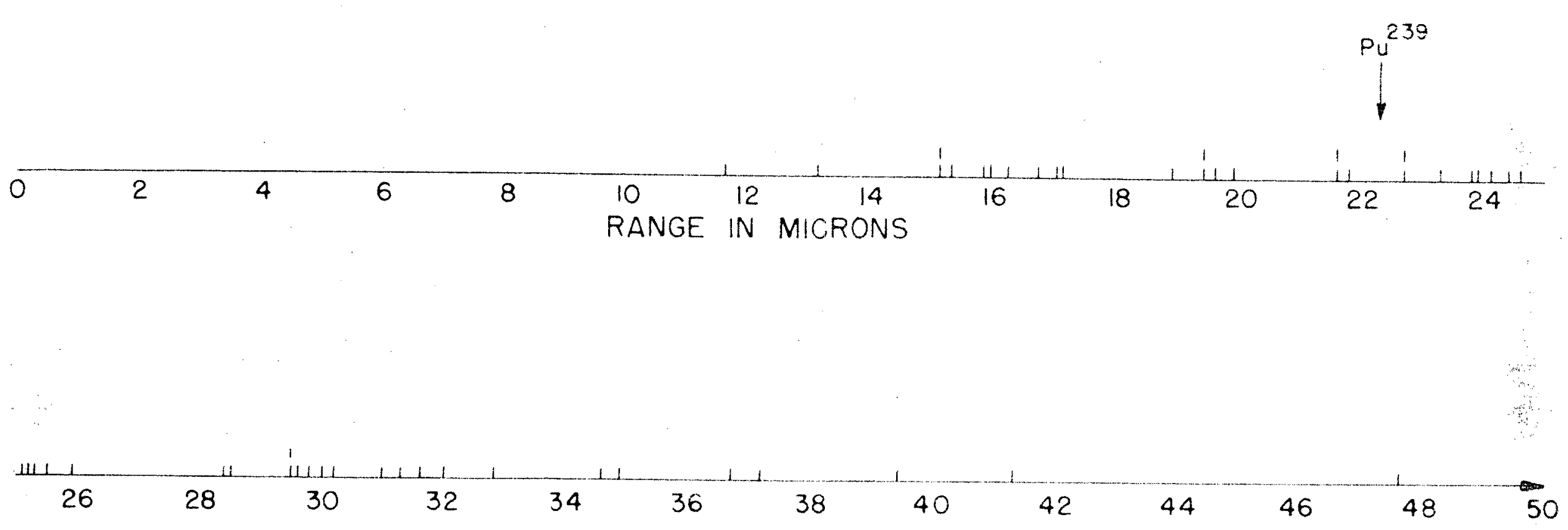

RANGE HISTOGRAM OF BLANK URINE SAMPLE

Figure-14 

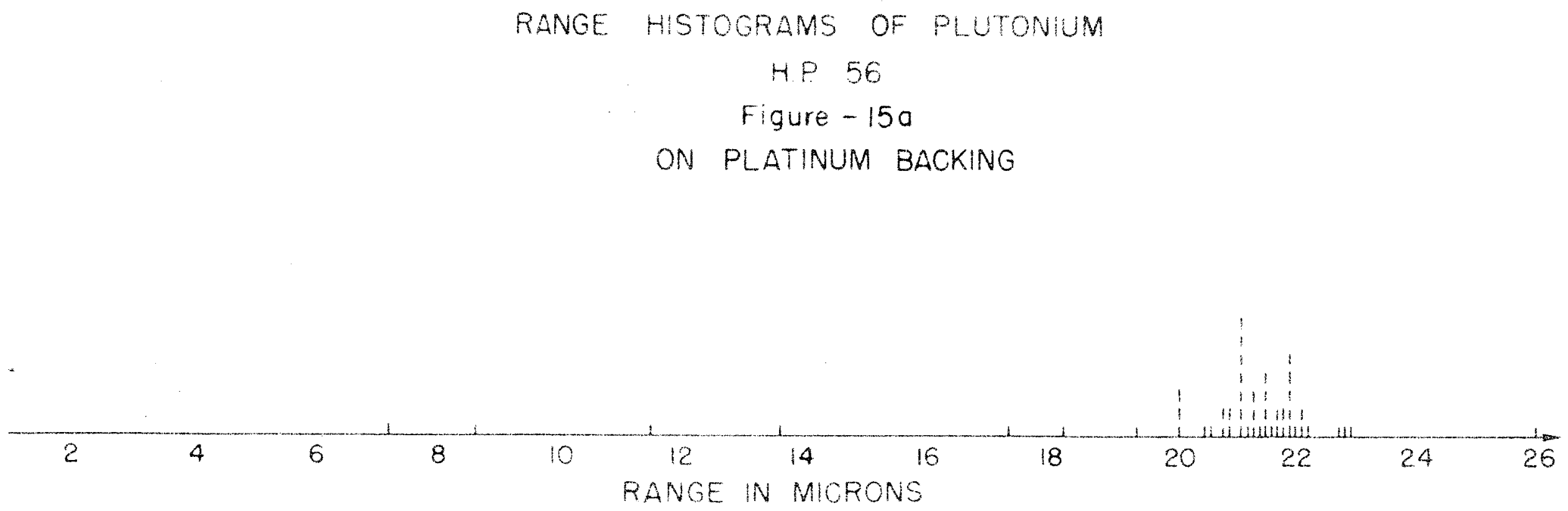

$$
\text { H.P } 57
$$$$
\text { Figure-15b }
$$

ON STAINLESS STEEL BACKING

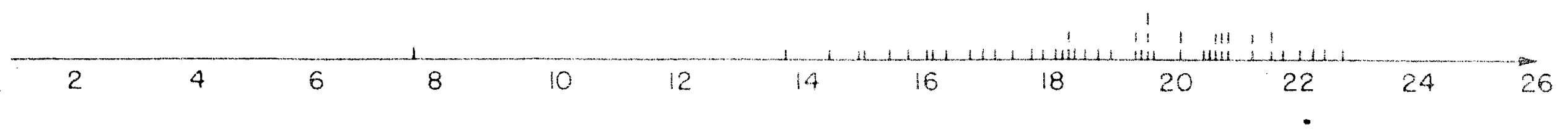


area only. In view of possible future automatic scanning techniques as outIined by Van der Grinten and Barker (loc。 cit.), it is also planned to produce a flat, circular deposit of the gramophone record type, using the same capillary tube and a heated turntable.

The method has already been of value in the solution of problems other than urinalysis. In March 1950 a contaminated paper was analyzed by the nuclear film technique. Although the alpha activity was high enough to be measured with a counter, the contaminant could not be identified without the use of a pulse analyzer, which was not available." On the other hand, exposure to a nuclear emulsion did yield the following results immediately: (a) the contaminant was radium, and (b) the contamination originated from a very few microscopic specks, located on the surface layer of the paper.

Figure 16 gives the range histogram of this contaminated paper. The absence of tracks between 30 and 35 microns and below 18 microns indicates that self-absorption was relatively unimportant and that consequently the activity was on the surface. 


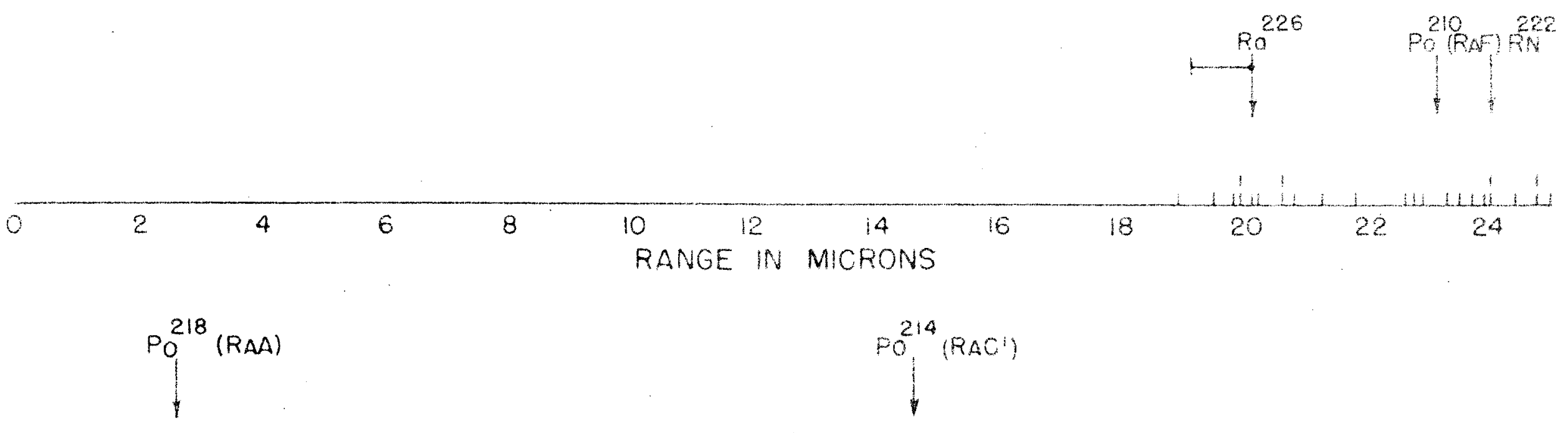

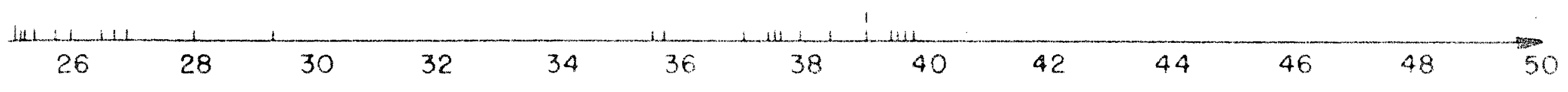


I. Gucker, Jro, F。To, $\mathrm{H}_{0} \mathrm{~B}_{0}$ Pickard, $\mathrm{C}$. To O Konski, and $J_{0} \mathrm{H}_{0}$ Pitts, $\mathrm{Jr}_{0}$ : OSRD-5501, August 1945

2. "Handbook on Aerosols", Atomic Energy Commission, Washington, D。Co, 1950

3. Hatch, To Fo: WASH-7, June 1949

4. LaMer, Vo $K$ 。 and Do Sinclair: OSRD-155, November 1941

5. Laskin, So: UR-129, August 1950

6. Toms, B。 A。: Porton Rep't No。 2251, JuIy 1943

7. Vonnegut, $B_{0}:$ GE Research Laboratory Report No。 RL-300, January 1950

8. Brown, J。Ho, K。 Mo Cook, E。 G。 Ney, and ToHatch: Am。J。Public Health 40, $450-459$ (1950)

9. Dallavalle, J。Mo: Micromeritics, 2nd Ed, Pitman Publishing Co., New York, 1948

10. Eyring, $\mathrm{H}_{0}:$ OSRD-292, December 1941

11. LaMer, V。K., D. Sinclair, D. E。 Goldman: NDRC-10.2-5 September 1943

12. LaMer, $V_{0} K_{0}$, et al。: OSRD-4904, April 1945

13. Ranz, W.E. and H. F. Johnstone: SO-1000, April 1950

14. Harrington, E。 Ro and W。 D. Crozier: NP-1091, December 1948

15. Lapple, C. E。: Chemical Engineer's Handbook, 3rd Ed。\& pp 1013-1050, MeGraw-Hill Book Co., New York, 1950

16. Fullam, E。 F。: GE Research Laboratory RL-202, April 1949

17. Roller, Po S。: J。 Franklin Inst。 223, 609-633 (1937)

18. Roller, P。S。: J。Phys。Chem。 45, 241-281 (1941)

19. Langmuir, I。: OSRD-865, September 1942

20. White, D。 Fo, I. Go Campbell, and D。 R。 Payne: Nature 166, 628-630 (1950)

21. Perkin, A。G。 and Pate, $L_{0}, J_{0}$ Chem。 Soc。67, 644 (1895)

22. Neelakantam, $K_{0}$ and Row, L。 Ro, Proc。IndoAcado Sci。, 14A, 307-312, (1941)

23. Bonner, J。 F。, Univo of Rochester, AEC Report UR-III 
24. Bonner, J.F., Ibid

25. Grinbaumowna and Marchlewski, Biochemische Zeitschrift, 290, P。261 (1937)

26. Sax, No I., "A Fluorophotometric Determination for Beryllium in Microquantities," General Electric Schenectady Works Laboratory

27. Neuman, W. F。, et al, J. of Biol. Chem., March 1948

28. Sandell, E. B., Ind, and Eng. Chem。, Anal. Ed., 12, 674-675 (1940)

29. SandeII, E. B., Ibid, 702-764 (1940)

30. Van der Grinten, Wo and D. F。 Barker, GE Research Laboratory Report, "The Detection of Weak Alpha Activities with Nuclear Emulsions." 